\title{
NuSTAR observations of water megamaser AGN
}

\author{
A. Masini ${ }^{1,2}$, A. Comastri ${ }^{1}$, M. Baloković ${ }^{3}$, I. Zaw ${ }^{4,5}$, S. Puccetti ${ }^{6,7}$, D. R. Ballantyne ${ }^{8}$, F. E. Bauer ${ }^{9,10,11,12,}$
} S. E. Boggs ${ }^{13}$, W. N. Brandt ${ }^{14,15,16}$, M. Brightman ${ }^{3}$, F. E. Christensen ${ }^{17}$, W. W. Craig ${ }^{13,18}$, P. Gandhi ${ }^{19,20}$, C. J. Hailey ${ }^{21}$, F. A. Harrison ${ }^{3}$, M. J. Koss ${ }^{22,26}$, G. Madejski ${ }^{23}$, C. Ricci ${ }^{9}, 12$, E. Rivers ${ }^{3}$, D. Stern ${ }^{24}$, and W. W. Zhang ${ }^{25}$

1 INAF-Osservatorio Astronomico di Bologna, via Ranzani 1, 40127 Bologna, Italy e-mail: alberto.masini4@unibo.it

2 Dipartimento di Fisica e Astronomia (DIFA), Università di Bologna, viale Berti Pichat 6/2, 40127 Bologna, Italy

3 Cahill Center for Astronomy and Astrophysics, California Institute of Technology, Pasadena, CA 91125, USA

4 New York University Abu Dhabi, PO Box 129188, Abu Dhabi, UAE

5 Center for Cosmology and Particle Physics, Department of Physics, New York University - Affiliate Member, 4 Washington Place, New York, NY 10003, USA

6 ASDC-ASI, via del Politecnico, 00133 Roma, Italy

7 INAF-Osservatorio Astronomico di Roma, via Frascati 33, 00040 Monte Porzio Catone, Italy

8 Center for Relativistic Astrophysics, School of Physics, Georgia Institute of Technology, Atlanta, GA 30332, USA

9 Instituto de Astrofísica, Facultad de Física, Pontificia Universidad Católica de Chile, 306 Santiago 22, Chile

10 Millennium Institute of Astrophysics, MAS, Nuncio Monseñor Sótero Sanz 100, Providencia, Santiago de Chile

11 Space Science Institute, 4750 Walnut Street, Suite 205, Boulder, Colorado 80301, USA

12 EMBIGGEN Anillo, Concepción, Chile

13 Space Science Laboratory, University of California, Berkeley, CA 94720, USA

14 Department of Astronomy and Astrophysics, 525 Davey Lab, The Pennsylvania State University, University Park, PA 16802, USA

15 Institute for Gravitation and the Cosmos, The Pennsylvania State University, University Park, PA 16802, USA

16 Department of Physics, 104 Davey Lab, The Pennsylvania State University, University Park, PA 16802, USA

17 DTU Space National Space Institute, Technical University of Denmark, Elektrovej 327, 2800 Lyngby, Denmark

${ }^{18}$ Lawrence Livermore National Laboratory, Livermore, CA 94550, USA

19 Centre for Extragalactic Astronomy, Department of Physics, University of Durham, South Road, Durham DH1 3LE, UK

20 School of Physics and Astronomy, University of Southampton, Highfield, Southampton SO17 1BJ, UK

21 Columbia Astrophysics Laboratory, Columbia University, New York, NY 10027, USA

22 Institute for Astronomy, Department of Physics, ETH Zurich, Wolfgang-Pauli-Strasse 27, 8093 Zurich, Switzerland

${ }^{23}$ Kavli Institute for Particle Astrophysics and Cosmology, SLAC National Accelerator Laboratory, Menlo Park, CA 94025, USA

24 Jet Propulsion Laboratory, California Institute of Technology, Pasadena, CA 91109, USA

25 NASA Goddard Space Flight Center, Greenbelt, MD 20771, USA

26 SNSF Ambizione Postdoctoral Fellow

Received 3 November 2015 / Accepted 9 February 2016

\section{ABSTRACT}

Aims. We study the connection between the masing disk and obscuring torus in Seyfert 2 galaxies.

Methods. We present a uniform X-ray spectral analysis of the high energy properties of 14 nearby megamaser active galactic nuclei observed by NuSTAR. We use a simple analytical model to localize the maser disk and understand its connection with the torus by combining NuSTAR spectral parameters with the available physical quantities from VLBI mapping.

Results. Most of the sources that we analyzed are heavily obscured, showing a column density in excess of $\sim 10^{23} \mathrm{~cm}^{-2}$; in particular, $79 \%$ are Compton-thick $\left(N_{\mathrm{H}}>1.5 \times 10^{24} \mathrm{~cm}^{-2}\right)$. When using column densities measured by NuSTAR with the assumption that the torus is the extension of the maser disk, and further assuming a reasonable density profile, we can predict the torus dimensions. They are found to be consistent with mid-IR interferometry parsec-scale observations of Circinus and NGC 1068. In this picture, the maser disk is intimately connected to the inner part of the torus. It is probably made of a large number of molecular clouds that connect the torus and the outer part of the accretion disk, giving rise to a thin disk rotating in most cases in Keplerian or sub-Keplerian motion. This toy model explains the established close connection between water megamaser emission and nuclear obscuration as a geometric effect.

Key words. masers - galaxies: active - galaxies: Seyfert

\section{Introduction}

There is strong evidence that a significant number of active galactic nuclei (AGN) are mildly or heavily obscured by a large amount of gas, preventing us from detecting their nuclear emission. The study of the column density distribution among Seyfert 2 galaxies (Risaliti et al. 1999) is a key element in understanding the nature and structure of the putative toroidal reprocessor of the AGN unified model (Antonucci 1993; Urry \& Padovani 1995), which is responsible for many of the observed differences between type 1 and type 2 Seyfert galaxies. The most common way to study the innermost regions of obscured AGN is through their hard X-ray emission, which can penetrate even very high obscuring column densities. 
The Nuclear Spectroscopic Telescope Array (NuSTAR) is a recent hard X-ray observatory launched in June 2012. It has two coaligned X-ray optics that focus X-ray photons onto two independent shielded focal plane modules (FPMs), namely FPMA and FPMB. Thanks to its focusing optics, it has a broad and high-quality spectral coverage from $3 \mathrm{keV}$ to $79 \mathrm{keV}$, a field of view (FoV) at $10 \mathrm{keV}$ of $10^{\prime} \times 10^{\prime}$, and an $18^{\prime \prime}$ FWHM with a half-power diameter of 58" (Harrison et al. 2013). Given these features, NuSTAR is suitable for studying the hard X-ray spectra of AGN with high sensitivity, distinguishing between the transmitted nuclear emission (i.e., radiation that penetrates the obscuring matter along the line of sight) and the scattered or reflected component (i.e., radiation that interacts with circumnuclear gas and gets absorbed or Compton-scattered). One of the NuSTAR scientific goals is to study the Compton-thick $\left(N_{\mathrm{H}}>1.5 \times 10^{24} \mathrm{~cm}^{-2}\right)$ AGN population, which is still poorly understood owing to the lack of good quality spectra above $10 \mathrm{keV}$. This class of obscured active nuclei (see Comastri 2004, for a review) is predicted from population synthesis models of the X-ray background (Gilli et al. 2007; Treister et al. 2009) to be a non-negligible contributor to the $\sim 30 \mathrm{keV}$ peak of the cosmic X-ray background (CXB), which is still today mostly unresolved (Civano et al. 2015; Mullaney et al. 2015; Aird et al. 2015; Harrison et al. 2015).

The $22 \mathrm{GHz}$ maser line emitted by water vapor molecules having the $6_{16}-5_{23}$ rotational transition can pass through thick absorbing matter and probe the innermost part of the nuclear structure, where high density and near edge-on geometry are needed to produce maser amplification. It has been a long time since the first water vapor extragalactic maser emission was discovered in M33 (Churchwell et al. 1977). Today, nearly 200 galaxies have been detected in $\mathrm{H}_{2} \mathrm{O}$ maser emission, some associated with disk structures, jets or outflows (e.g., see Table 1 of Lo 2005). Because of their high luminosities with respect to Galactic masers, extragalactic water masers associated to AGN are generally called megamasers, while those associated to starforming regions are sometimes referred to as kilomasers.

Very long baseline interferometry (VLBI) radio observations provide a robust tool for studying subparsec structures. In particular, in disk maser systems, precise estimates of the central dynamical mass can be performed. VLBI maser mapping has been used to test the existence of supermassive black holes (SMBHs), ruling out other candidates (such as rich clusters of stars), and to measure distances independently of a cosmological model (see, e.g., Reid et al. 2009, 2013). Notably, megamasers tend to be found in Seyfert 2 (Sy2) galaxies and, in particular, in Comptonthick ones (Greenhill et al. 2008), which according to the AGN unification scheme, are likely to be those where the obscuring structure is seen nearly edge-on.

High-quality hard X-ray ( $>10 \mathrm{keV})$ data coupled with highresolution radio maps of the nuclear emission allow new studies of the physics of obscured AGN. Some previous work concentrated on the connection between masing activity and high obscuring column densities in active nuclei, identifying some general and phenomenological results (Greenhill et al. 2008; Castangia et al. 2013). However, many questions are still unanswered. Physical conditions, such as the temperature, density, and pressure of matter in the vicinity of the SMBH, are still uncertain. It is not completely clear whether the maser emission is associated with the outer part of the accretion disk or if it is part of the toroidal structure obscuring the nucleus along our line of sight. In this paper we first present new spectral analyses of NuSTAR observations of megamaser sources, using a sample of local AGN with good quality X-ray data and radio maps.
We then combine the information from the hard X-ray and radio bands to derive a physical picture of the complex environment in which SMBHs are growing.

The paper is organized as follows. In Sect. 2 we present the megamaser sample and the X-ray analyses with a brief explanation of modeling and some notes on individual sources. Section 3 presents results we obtained by combining the spectral and maser disk parameters with a toy model. A discussion of the toy model is given in Sect. 4. We give a summary in Sect. 5.

\section{Data and spectral analysis}

\subsection{The sample}

To build up a sample of disk megamaser sources with highquality maser maps, precise black hole mass estimates, and hard $\mathrm{X}$-ray spectral coverage, we cross-correlated a list of VLBImapped water megamasers from the Megamaser Cosmology Project $^{1}$ (MCP, see Henkel et al. 2012) with NuSTAR observations and well known disk maser sources studied in the literature. We found 11 objects. We then enlarged the sample by adding three more sources with VLBI radio maps available, but lacking NuSTAR data (refer to Castangia et al. 2013, for X-ray and maser disk properties of these). The total sample is then composed of 14 sources, which are all the disk water megamasers known today with both precise VLBI maps and hard X-ray spectra. Their main properties are listed in Table 1. However, we emphasize that this is not a complete sample of all the water megamasers known today, which can be found in Pesce et al. (2015).

\subsection{Data reduction}

We present NuSTAR hard X-ray spectral results for 11 sources. In particular, we use archival data for NGC 1194, NGC 1386, NGC 2273, NGC 2960, NGC 3079, NGC 3393, NGC 4388, and IC 2560, for which observation dates and exposure times can be found in Table 2. For NGC 4945, NGC 1068, and the Circinus galaxy, spectral parameters are taken from Puccetti et al. (2014), Bauer et al. (2015), and Arévalo et al. (2014), respectively.

The raw events files were processed using the NuSTAR Data Analysis Software package v. 1.4.1 (NuSTARDAS) ${ }^{2}$. Calibrated and cleaned event files were produced using the calibration files in the NuSTAR CALDB (20150225) and standard filtering criteria with the nupipeline task. We used the nuproducts task included in the NuSTARDAS package to extract the NuSTAR source and background spectra using the appropriate response and ancillary files. We extracted spectra and light curves in each focal plane module using circular apertures of different radii, aimed at optimizing the signal-to-noise ratio at high energies for every source (see Baloković et al., in prep., for further details). Background spectra were extracted using source-free regions on the same detector as the source. All spectra were binned to a minimum of 20 photons per bin using the HEAsoft task grppha.

\subsection{Spectral analysis}

The spectral analysis was carried out using the XSPEC software (Arnaud 1996). We started by fitting the spectra with simple

\footnotetext{
1 http://safe.nrao.edu/wiki/bin/view/Main/ PublicWaterMaserList

2 http://heasarc.gsfc.nasa.gov/docs/nustar/analysis/ nustar_swguide.pdf
} 
Table 1. Megamaser sample, global properties, and references.

\begin{tabular}{|c|c|c|c|c|c|c|c|c|c|}
\hline Name & (2) & $\begin{array}{c}N_{\mathrm{H}} \\
{\left[10^{24} \mathrm{~cm}^{-2}\right]} \\
(3)\end{array}$ & $\begin{array}{c}\log \left(L_{2-10}\right) \\
{\left[\operatorname{erg~s}^{-1}\right]} \\
(4)\end{array}$ & (5) & $\begin{array}{c}M_{\mathrm{BH}} \\
{\left[10^{6} M_{\odot}\right]} \\
(6)\end{array}$ & (7) & $\begin{array}{c}R_{\mathrm{d}} \\
{[\mathrm{pc}]} \\
(8)\end{array}$ & $\begin{array}{c}\text { Disk size } \\
{[\mathrm{pc}]} \\
(9)\end{array}$ & (10) \\
\hline NGC 1068 & 0.0038 & $>5.6$ & 43.34 & Bau14 & $8.0 \pm 0.3$ & Lod03 & 0.27 & $0.65-1.1$ & Gre97 \\
\hline NGC 1194 & 0.0136 & $1.4_{-0.2}^{+0.3}$ & 42.78 & this work & $65 \pm 3$ & Kuo11 & 0.14 & $0.54-1.33$ & Kuo11 \\
\hline NGC 1386 & 0.0029 & $5 \pm 1$ & 41.90 & this work & $1.2_{-06}^{+1.1}$ & $\mathrm{McC} 13^{\dagger}$ & 0.05 & $0.44-0.94$ & Til08 \\
\hline NGC 2273 & 0.0061 & $>7.3$ & 43.11 & this work & $7.5 \pm 0.4$ & Kuo11 & 0.20 & $0.034-0.20$ & this work* \\
\hline NGC 2960 & 0.0165 & $0.5_{-0.3}^{+0.4}$ & 41.41 & this work & $11.6 \pm 0.5$ & Kuo11 & 0.03 & $0.13-0.37$ & Kuo11 \\
\hline NGC 3079 & 0.0037 & $2.5 \pm 0.3$ & 42.15 & this work & $2.4_{-1.2}^{+2.4}$ & $\mathrm{McC}_{13}{ }^{\dagger}$ & 0.07 & $0.4-1.3$ & Kon05 \\
\hline NGC 3393 & 0.0125 & $2.2_{-0.2}^{+0.4}$ & 43.30 & this work & $31 \pm 2$ & Kon08 & 0.25 & $0.17-1.5$ & Kon08 \\
\hline NGC 4388 & 0.0084 & $0.44 \pm 0.06$ & 42.59 & this work & $8.5 \pm 0.2$ & Kuo11 & 0.11 & $0.24-0.29$ & Kuo11 \\
\hline NGC 4945 & 0.0019 & $3.5 \pm 0.2$ & 42.52 & Puc14 & $1.4_{-0.5}^{+0.7}$ & $\mathrm{McC}_{13}{ }^{\dagger}$ & 0.10 & $0.13-0.41$ & this work ${ }^{* *}$ \\
\hline IC 2560 & 0.0098 & $>6.7$ & 42.98 & this work & $3.5 \pm 0.5$ & Yam12 & 0.17 & $0.087-0.335$ & Yam12 \\
\hline Circinus & 0.0015 & $8.7 \pm 1.5$ & 42.57 & Are14 & $1.7 \pm 0.3$ & Gre03 & 0.11 & $0.11-0.4$ & Gre03 \\
\hline NGC 4258 & 0.0015 & $0.087 \pm 0.003$ & 41.2 & Cas13 & $39 \pm 3$ & Til08 & 0.02 & $0.12-0.28$ & Til08 \\
\hline NGC 6264 & 0.0340 & $>1$ & 42.6 & Cas 13 & $29.1 \pm 0.4$ & Kuo11 & 0.11 & $0.24-0.80$ & Kuo11 \\
\hline UGC 3789 & 0.0109 & $>1$ & 42.3 & Cas 13 & $10.4 \pm 0.5$ & Kuo11 & 0.08 & $0.084-0.30$ & Kuo11 \\
\hline
\end{tabular}

Notes. Main properties of the disk maser AGN sample used in this work. The last three sources are the ones lacking NuSTAR data.(1) - Galaxy name. (2) - Redshift. (3) - Best fit intrinsic column density. (4) - Logarithm of the best fit intrinsic (deabsorbed) 2-10 keV luminosity. (5) References for Cols. (3)-(4): Are14 - Arévalo et al. (2014); Bau14 - Bauer et al. (2015); Cas13 - Castangia et al. (2013); Puc14 - Puccetti et al. (2014). (6) - AGN central mass. (7) - References for Col. (6): Gre03 - Greenhill et al. (2003); Til08 - Tilak et al. (2008); Kon08 - Kondratko et al. (2008); Kuo11 - Kuo et al. (2011); Lod03 - Lodato \& Bertin (2003); McC13 - McConnell \& Ma (2013); Yam12 - Yamauchi et al. (2012). (8) - Dust sublimation radius, calculated using the relation from Gandhi et al. (2009). See Sect. 3 for details. (9) - Maser disk inner and outer radii. (10) - References for Col. (9): Gre97 - Greenhill \& Gwinn (1997); Gre03 - Greenhill et al. (2003); Kon05 - Kondratko et al. (2005); Kon08 Kondratko et al. (2008); Kuo11 - Kuo et al. (2011); Til08 - Tilak et al. (2008); Yam12 - Yamauchi et al. (2012). ${ }^{(*)}$ New maser disk extension estimate from VLBI maps. ${ }^{(* *)}$ Adapted from Greenhill et al. (1997). ${ }^{(\dagger)}$ Maser method mass for which in the original paper an uncertainty is not provided. The error given by McConnell \& Ma (2013) is overestimated.

power law models for an initial visual inspection of the broadband spectral curvature and X-ray absorption. We then applied phenomenological models, such as plcabs (Yaqoob 1997) or pexrav (Magdziarz \& Zdziarski 1995), to model the hard X-ray continua. The former describes X-ray transmission of an intrinsic power law with an exponential cutoff through an obscuring medium, taking the effects of Compton scattering into account. The latter models Compton reflection on a slab of neutral material with infinite optical depth.

It was always possible to find a combination of these two models that gave an excellent fit to the data. However, as pointed out by Murphy \& Yaqoob (2009), using plcabs and pexrav may produce a bias toward fits dominated by the direct continuum. These initial results, then, need to be tested against more self-consistent and physically motivated models based on Monte Carlo simulations, such as MYTorus (Murphy \& Yaqoob 2009) and Torus (Brightman \& Nandra 2011). They both model the hard X-ray spectrum emitted through a toroidal reprocessor, consisting of a transmitted continuum (photons passing through the torus without interacting), a scattered or reflected component that is made up of photons that interact with matter via Compton scattering, and emission lines (mostly iron $\mathrm{K} \alpha$ and $\mathrm{K} \beta$ ). MYTorus allows a dynamic decoupling of these three components to simulate different geometries. It can be used in the default configuration ("MYTorus coupled"), modeling a classical "donut-shaped" toroidal reprocessor with a fixed covering factor of 0.5 (i.e., the half-opening angle $\theta_{\text {tor }}$ of the torus is $60^{\circ}$, measured as the angle between the axis of the system and the edge of the torus itself), or in a more complex way, called "MYTorus decoupled".
In this configuration, part of the reflection from the inner far side of the reprocessor could be unobscured by material on the near side of it. In this case, the far-side reflection, at least below $\sim 10 \mathrm{keV}$, can dominate the observed spectrum. This backreflected continuum and the associated lines are parameterized with a MYTorus face-on reflection spectrum, obtained by fixing the inclination angle of the system $\theta_{\mathrm{obs}}$ to $0^{\circ}$. On the other hand, the forward-scattered emission and associated emission lines are approximated using a MYTorus edge-on reflection spectrum, obtained by fixing $\theta_{\text {obs }}$ to $90^{\circ}$. The relative strength of these two components (front and back-scattered) is encoded in two constants, namely $A_{S 90}$ and $A_{S 00}$, which are left free to vary. Their respective line components for this geometry are normalized with $\mathrm{A}_{\mathrm{L} 90}$ and $\mathrm{A}_{\mathrm{L} 00}$. Finally, in the most general case, the column density $N_{\mathrm{H}}$ obscuring the direct continuum can be decoupled from the column density responsible for the back reflection or the forward reflection or both. A single $N_{\mathrm{H}}$ value is adopted for the sake of simplicity. We refer to Yaqoob (2012) for an exhaustive example of the use of the model in its decoupled mode. The Torus model does not decouple the three components, but has the opening angle as a free parameter, allowing the measurement of the covering factor (see Brightman et al. 2015). In the following, we always assume a nearly edge-on inclination of the reprocessor (i.e., we fix the inclination angle of the system, $\theta_{\text {obs }}$, to $90^{\circ}$ ), even if the toroidal geometry is slightly different between the two models.

We first apply all these physical models alone, then we concentrate on the best among them and refine it adding other line features, if needed, or another power law. The last is usually significant at lower energies (below $\sim 5 \mathrm{keV}$ ) and is thought to be 
Table 2. NuSTAR observation details for the eight sources analyzed.

\begin{tabular}{lcc}
\hline \hline Name & Date of observation & $\begin{array}{c}\text { Exposure time } \\
{[\mathrm{ks}]}\end{array}$ \\
\hline NGC 1194 & 2015-Feb.-28 & 31 \\
NGC 1386 & 2013-Jul.-19 & 21 \\
NGC 2273 & 2014-Mar.-23 & 23 \\
NGC 2960 & 2013-May-10 & 21 \\
NGC 3079 & 2013-Nov.-12 & 21 \\
NGC 3393 & 2013-Jan.-28 & 15 \\
NGC 4388 & 2013-Dec.-27 & 21 \\
IC 2560 & 2013-Jan.-28, 2014-Jul.-16 & 73 \\
\hline
\end{tabular}

due to electron scattering in an ionized zone extended on a size scale larger than the obscuring structure, even if its contribution depends on modeling details. To model this physical situation in a simple manner, we tied all the power law parameters to the primary one (i.e., photon index, redshift, normalization) and multiplied it by a constant, namely $f_{\mathrm{s}}$, which is a free parameter in the fit that quantifies the fraction of the primary power law scattered at low energies. We refer to this power law as a "scattered power law". We explain in detail the general fitting procedure for NGC 1194, while for the remaining eight sources we summarize the most relevant findings, in particular the most precise measurement of the column density available to date. Errors quoted always refer to $90 \%$ confidence limits for one interesting parameter, if not stated otherwise.

\subsubsection{NGC 1194}

NGC 1194 is a nearby Seyfert 1.9 galaxy. It hosts a circumnuclear maser disk, which allowed a precise measurement of the BH mass of $(6.5 \pm 0.3) \times 10^{7} M_{\odot}$ (Kuo et al. 2011). Fitting the spectrum with an absorbed power law using a Galactic column returns an uncharacteristically hard photon index $(\Gamma \sim$ 0.5 ) and leaves large residuals, in particular a prominent line feature at $\sim 6-7 \mathrm{keV}$ and an excess between 10 and $30 \mathrm{keV}$ $\left(\chi^{2} / v=494 / 117\right)$. These are typical spectral signatures of an obscured AGN. A plcabs model (which accounts for obscuration) with two intrinsically narrow $(\sigma=10 \mathrm{eV})$ Gaussian components for the lines at $6-7 \mathrm{keV}$ returns a much better fit $\left(\chi^{2} / v=186 / 112\right)$. The obscuration is in the Compton-thin regime $\left(N_{\mathrm{H}} \sim 6 \times 10^{23} \mathrm{~cm}^{-2}\right)$, and the photon index is $\sim 1$. The residuals still show a hump at $\sim 20 \mathrm{keV}$ and signatures of soft excess at energies $<5 \mathrm{keV}$. A better fit is obtained if plcabs is replaced by a pexrav model $\left(\chi^{2} / v=96 / 113, \Gamma \sim 1.6\right)$. Using both models returns an even better fit with $\Gamma \sim 1.6$ and $N_{\mathrm{H}} \sim 10^{24} \mathrm{~cm}^{-2}\left(\chi^{2} / v=85 / 111\right)$, in which the plcabs component is still significant at more than $99 \%$ confidence limit. This appears to be an unphysical situation, since the flux in the reflected component is much greater than the total intrinsic flux of the source.

We then apply more physically self-consistent models. An almost edge-on Torus model with a fixed opening angle $\left(\theta_{\text {tor }}=\right.$ $\left.60^{\circ}\right)$ returns an unacceptable fit $\left(\chi^{2} / v=220 / 116\right)$, with $\Gamma \sim 1.4$ and $N_{\mathrm{H}} \sim 7 \times 10^{23} \mathrm{~cm}^{-2}$. The fit can be improved by fitting for the torus opening angle $\left(\chi^{2} / v=201 / 116\right)$, which has a best fit value of $\theta_{\text {tor }}=26^{\circ}$ (the lower limit accepted by the model) with $\Gamma \sim 1.4$ and $N_{\mathrm{H}} \sim 6 \times 10^{23} \mathrm{~cm}^{-2}$. A MYTorus model in its default configuration (i.e., coupled mode) returns a similar fit to the Torus one: $\chi^{2} / v=217 / 116, \Gamma \sim 1.4$ and $N_{\mathrm{H}} \sim 6 \times 10^{23} \mathrm{~cm}^{-2}$. A common feature of these models is the underprediction of both the flux of the line component at 6-7 keV (see, e.g., Vasylenko et al. 2015) and the emission below $5 \mathrm{keV}$. A MYTorus model in its decoupled mode is then applied $\left(\chi^{2} / v=161 / 115\right)$. The front-scattered component vanishes, the photon index is $\sim 1.6$, and the column density is $\sim 10^{24} \mathrm{~cm}^{-2}$. These values are consistent with what is found by applying phenomenological models in the first part of the analysis. We note that scattering $\sim 3 \%$ of the primary continuum into a scattered power law and adding a line feature at $(6.8 \pm 0.1) \mathrm{keV}$ do not change the fundamental fit parameters, but improve it at more than $99 \%$ confidence limit $\left(\chi^{2} / v=113 / 112, \Delta \chi^{2} / \Delta v=48 / 3\right)$. Even if the best fit model is made up of a combination of plcabs, pexrav, and zgauss models $\left(\chi^{2} / v=85 / 111\right)$, we choose to rely on the best fit among the self-consistent ones, as for the other sources, which is the decoupled MYTorus model (see Fig. 1a).

In summary, given that phenomenological models point toward a highly obscured, reflection-dominated source and that the best fit in a physical and self-consistent model is represented by a back-scattered radiation-dominated MYTorus model, we conclude that NGC 1194 is a Compton-thick AGN with the column density $N_{\mathrm{H}}=1.4_{-0.2}^{+0.3} \times 10^{24} \mathrm{~cm}^{-2}$, consistent with the one reported by Greenhill et al. (2008). We note that, according to the best fit model, reflection only dominates below $\sim 10 \mathrm{keV}$. Best fit spectral parameters are given in Table 3.

\subsubsection{NGC 1386}

NGC 1386 hosts a water maser source (Braatz et al. 1997), although it is not clear whether the maser spots trace a rotating thin disk or align in front of an underlying continuum (i.e., are jet masers). This makes the central black hole mass estimate challenging so we adopted the one reported by McConnell \& $\mathrm{Ma}$ $(2013)^{3}$, which is $1.2_{-0.6}^{+1.1} \times 10^{6} M_{\odot}$. A default MYTorus model fails $\left(\chi^{2} / v=131 / 27\right)$, and two statistically indistinguishable sets of parameters are possible: one with a Compton-thin obscuration $\left(N_{\mathrm{H}} \sim 6 \times 10^{23} \mathrm{~cm}^{-2}\right)$ and $\Gamma \sim 1.4$, and one with a severely obscured AGN $\left(N_{\mathrm{H}} \sim 10^{25} \mathrm{~cm}^{-2}\right)$ and $\Gamma \sim 2.6$. A decoupled MYTorus model points toward a back-scattered radiationdominated spectrum with the same parameters as for the coupled case $\left(\chi^{2} / v=65 / 25\right)$. The unacceptable fit $\left(\chi^{2} / v=57 / 27\right)$ of the Torus model arises from an underestimation of the line contribution and provides the best $\chi^{2}$ among the physical models. Following Brightman et al. (2015), we added a line component to the fit at $(6.5 \pm 0.1) \mathrm{keV}$ and get $\chi^{2} / v=32 / 25$. A scattered power law is not required by the data (Fig. 1b). The central source is then obscured by Compton-thick material of column density $N_{\mathrm{H}}=(5 \pm 1) \times 10^{24} \mathrm{~cm}^{-2}$. Best fit parameters are given in Table 3. We note that our results agree with Brightman et al. (2015), where they focused on the Torus model for a covering factor estimate.

\subsubsection{NGC 2273}

The mass of the SMBH nested in the barred spiral galaxy NGC 2273 was measured by Kuo et al. (2011) to be $M_{\mathrm{BH}}=$ $(7.5 \pm 0.4) \times 10^{6} M_{\odot}$. The reported parameters put the maser disk very close to the central engine $(0.028-0.084 \mathrm{pc})$. This makes NGC 2273 an outlier in some relations (Castangia et al. 2013). To determine whether it is truly an outlier or whether emission at larger radii is missed by the VLBI observations, we derived radii from the more sensitive single-dish spectra taken with the

http://blackhole.berkeley.edu/ 


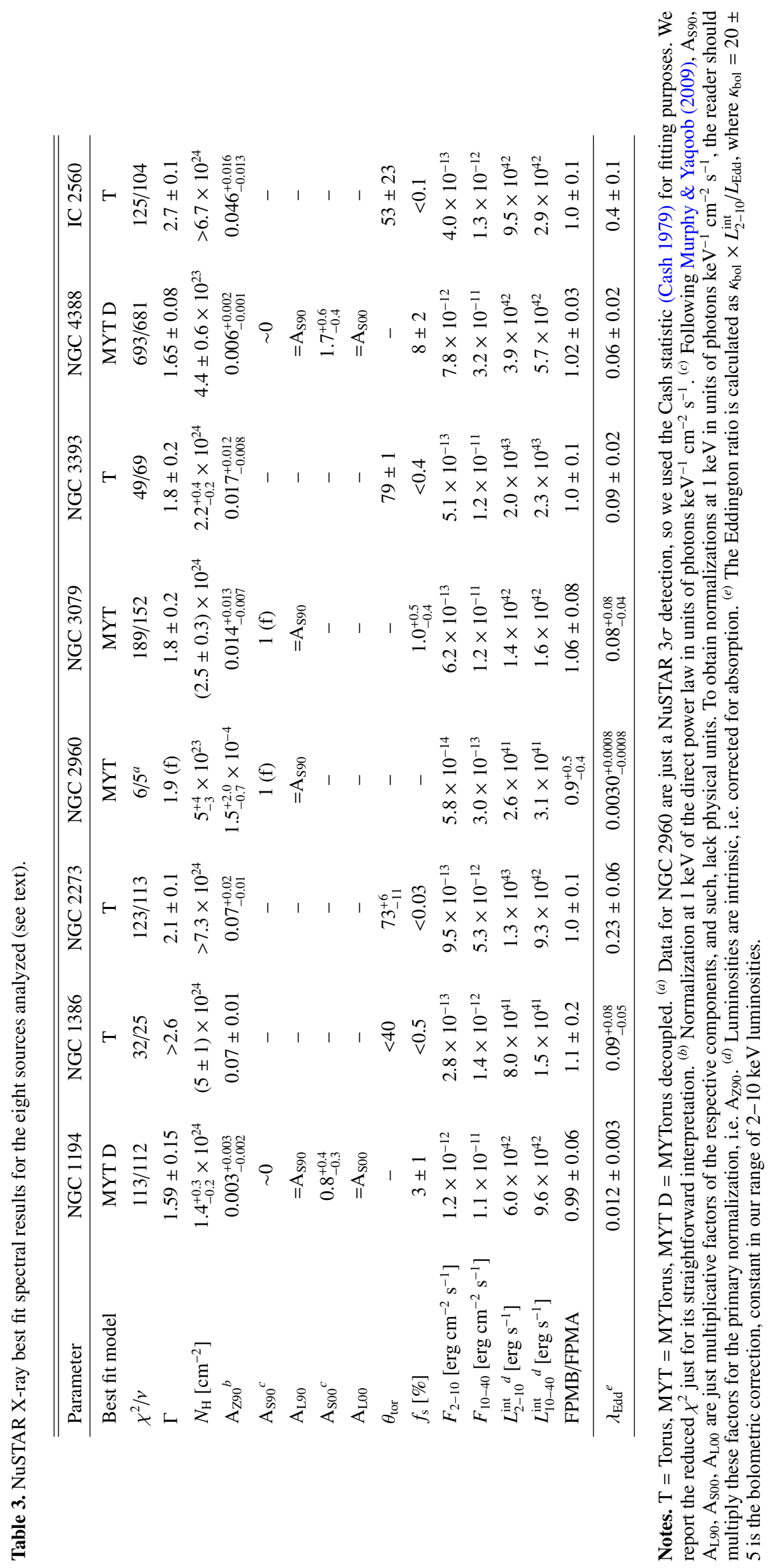




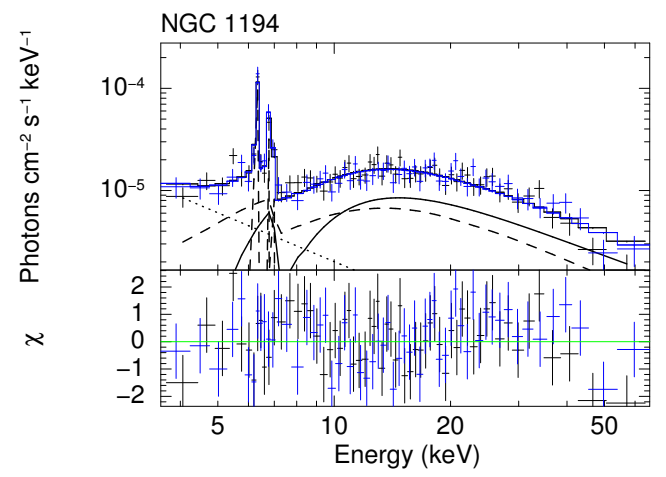

(a) MYT decoupled + zpow + zgauss

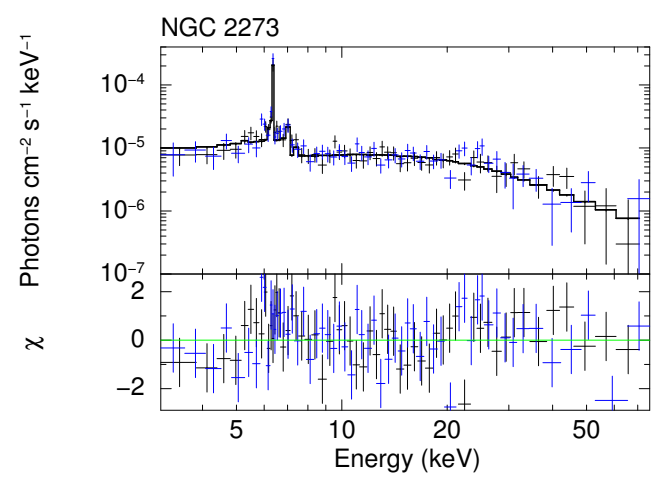

(c) Torus

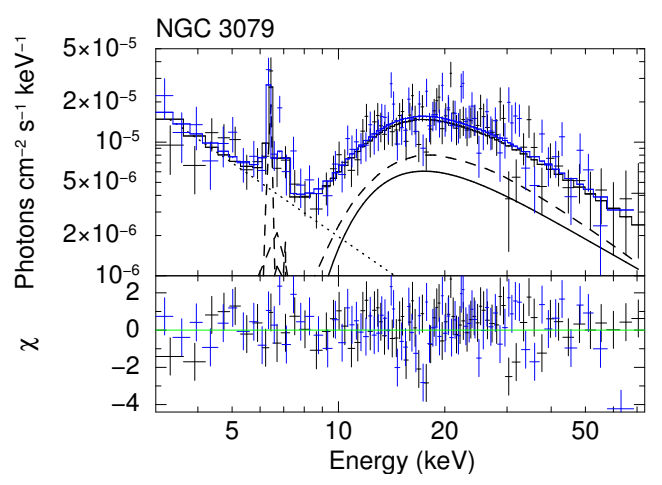

(e) MYTorus + zpow

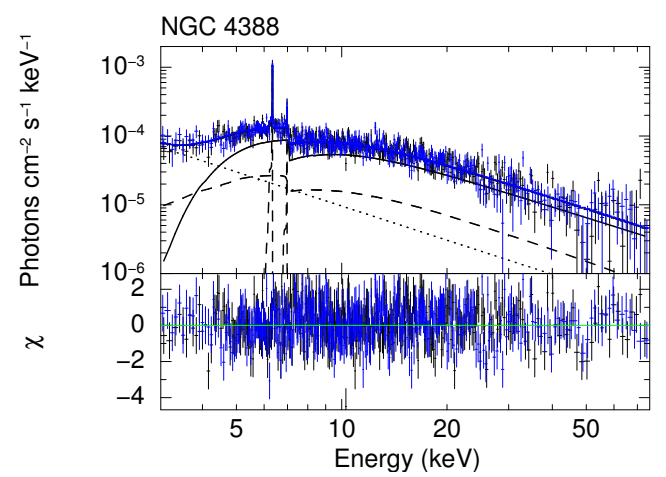

(g) MYT decoupled + zpow

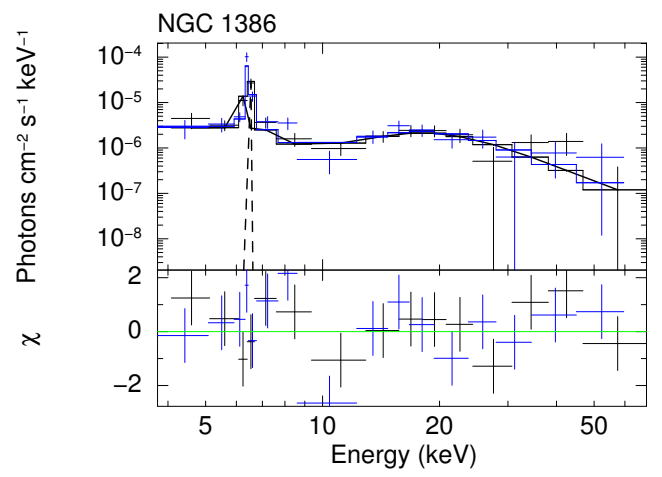

(b) Torus + zgauss

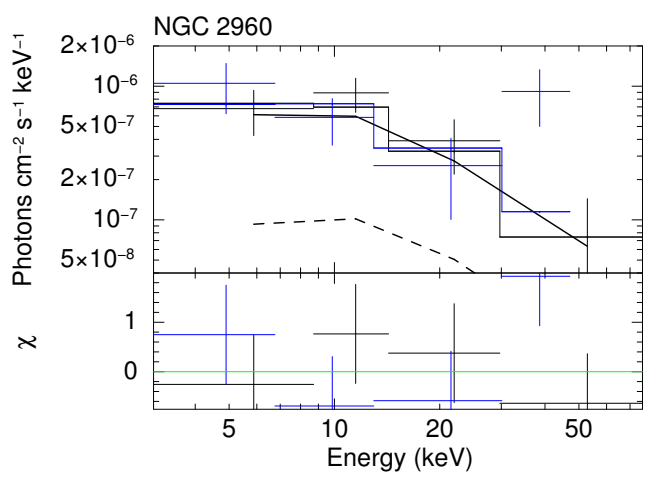

(d) MYTorus

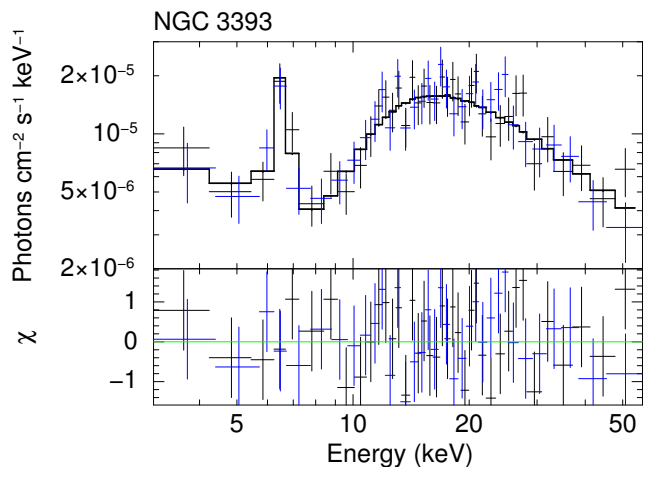

(f) Torus

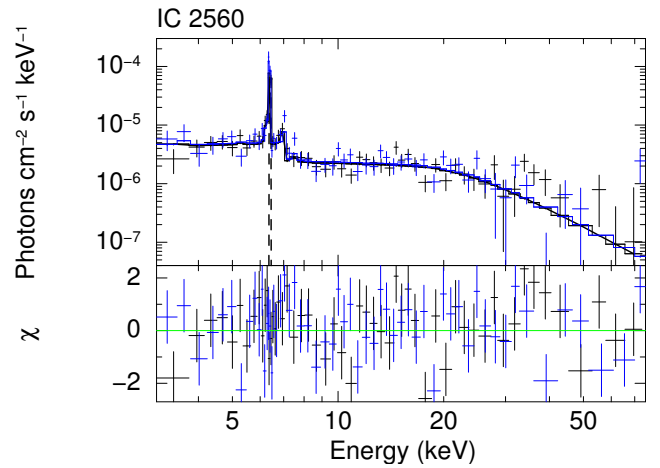

(h) Torus + zgauss

Fig. 1. NuSTAR spectra, best-fitting models and residuals for the eight sources analyzed. FPMA data are shown in black, while FPMB ones are shown in blue. When adopting a MYTorus model, the solid line represents the primary continuum. The reflection and line components are shown as the dashed line, while the scattered power law is shown as the dotted line. When adopting the Torus model, the solid line represents the total spectrum, while add-on line components are shown as the dashed line. 
Green Bank Telescope (Kuo et al. 2011). To do so, we assumed a systemic velocity of $1840 \mathrm{~km} \mathrm{~s}^{-1}$ (from NED ${ }^{4}$ ), a SMBH mass of $7.5 \times 10^{6} M_{\odot}$ (Kuo et al. 2011), and Keplerian rotation (Kuo et al. 2011). From the highest and lowest velocity emission (we require the emission to be at least 5 times the rms) of the highvelocity maser features, we find that the innermost radius is $\sim 0.034$ pc, consistent with Kuo et al. (2011), but that the outermost radius is $\sim 0.2 \mathrm{pc}$. We adopt these values in the following analysis.

A MYTorus model cannot account for the line emission, and it gives an unacceptable fit $\left(\chi^{2} / v=267 / 113\right)$. Its decoupled mode provides an acceptable fit $\left(\chi^{2} / v=131 / 111\right)$, pointing toward a back-scattered, reflection-dominated AGN. However, the best fit is found with the Torus model, where the source is heavily Compton-thick and a lower limit on the column density is found $\left(N_{\mathrm{H}}>7.3 \times 10^{24} \mathrm{~cm}^{-2}\right)$, consistently with Guainazzi et al. (2005) and Awaki et al. (2009). Torus spectral parameters are found in Table 3, while the best fit model is shown in Fig. 1c.

\subsubsection{NGC 2960}

The central black hole mass of the spiral megamaser galaxy NGC 2960 (Mrk 1419) as reported by Kuo et al. (2011) is $(1.16 \pm 0.05) \times 10^{7} M_{\odot}$. NGC 2960 is very faint, and a $21 \mathrm{ks}$ NuSTAR snapshot resulted in poor quality data that prevented us from significantly constraining spectral parameters. The fitting procedure in XSPEC was carried out using the Cash statistic (Cash 1979), but we report the reduced $\chi^{2}$ for direct comparison with other sources. Using a default MYTorus model and fixing the photon index $\Gamma=1.9$ (Fig. 1d), the fit returns an obscured, but Compton-thin, source $\left(N_{\mathrm{H}}=5_{-3}^{+4} \times 10^{23} \mathrm{~cm}^{-2}\right)$, which is marginally consistent with Greenhill et al. (2008). Results are listed in Table 3.

\subsubsection{NGC 3079}

The low ionization nuclear emission-line region (LINER) galaxy NGC 3079 presents a thick, flared, probably star-forming and self-gravitating maser disk (Kondratko et al. 2005). The disk's outer radius is indeed beyond the sphere of influence radius of the central mass, which is $2.4_{-1.2}^{+2.4} \times 10^{6} M_{\odot}$ (McConnell \& Ma 2013). Either Torus $\left(\chi^{2} / v=193 / 152\right)$ or MYTorus $\left(\chi^{2} / v=\right.$ $189 / 152)$, both with a scattered power law dominating below $5 \mathrm{keV}$, give similar results. Using a decoupled MYTorus model $\left(\chi^{2} / v=189 / 150\right)$, the back-scattered contribution vanishes, confirming that the source is dominated by reflection below $10 \mathrm{keV}$. We therefore chose the coupled MYTorus model as the best fit (Fig. 1e) and conclude that NGC 3079 is transmissiondominated with a column density of $N_{\mathrm{H}}=(2.5 \pm 0.3) \times 10^{24} \mathrm{~cm}^{-2}$ (see Table 3 for other parameters). This result agrees with the one found by Brightman et al. (2015) using the Torus model.

\subsubsection{NGC 3393}

The nearby barred galaxy NGC 3393 presents an edge-on maser disk that allowed Kondratko et al. (2008) to measure the central mass to be $(3.1 \pm 0.2) \times 10^{7} M_{\odot}$. An excellent fit is found with a Torus model $\left(\chi^{2} / v=49 / 69\right)$, with parameters reported in Table 3 . This fit is formally indistinguishable from a MYTorus model with a scattered power law in the soft part

\footnotetext{
4 http://ned.ipac.caltech.edu/
}

of the spectrum, either coupled or decoupled $\left(\chi^{2} / v=49 / 69\right.$ and $\chi^{2} / v=48 / 67$, respectively), but we chose Torus as the best fit because it only requires one component (i.e., the scattered power law is not significant, see Fig. 1f). However, the spectral parameters are the same within the uncertainties. We therefore conclude that NGC 3393 hosts a Compton-thick AGN $\left(N_{\mathrm{H}}=2.2_{-0.2}^{+0.4} \times 10^{24} \mathrm{~cm}^{-2}\right)$, in agreement with the results of Koss et al. (2015).

\subsubsection{NGC 4388}

The Virgo cluster member NGC 4388 hosts an active SMBH of mass $(8.5 \pm 0.2) \times 10^{6} M_{\odot}$ (Kuo et al. 2011). In their paper, Kuo et al. (2011) suggest using this mass value with caution because of the lack of systemic maser activity and the inability to robustly assess the Keplerian motion of the maser spots. Among the self-consistent models (Torus, MYTorus coupled, MYTorus decoupled) the last gives the best $\chi^{2}$, although spectral parameters are consistent among them all. A Torus model points toward smaller opening angles (i.e., larger covering factor, $\sim 0.9$ ), which could account for the line emission $\left(\chi^{2} / v=761 / 684\right)$. Fitting with MYTorus in coupled mode underestimates the line feature $\left(\chi^{2} / v=803 / 684\right)$. This could be due to supersolar iron abundance, a broad range of $N_{\mathrm{H}}$ with different covering factors, or to a covering factor greater than that of the model, as suggested by the Torus model. Decoupling MYTorus does not change the general results, while the back-scattered radiation seems to be favored over the vanishing front-scattered one $\left(\chi^{2} / v=729 / 682\right)$. The fit can be improved by adding a scattered power law below $5 \mathrm{keV}$, which brings the reduced chi-squared to $\chi^{2} / v=693 / 681$ (Fig. $1 \mathrm{~g}$ ). According to the best fit model, NGC 4388 is a Compton-thin $\left(N_{\mathrm{H}}=4.2 \pm 0.5 \times 10^{23} \mathrm{~cm}^{-2}\right)$ transmission-dominated source, and our results agree with the constraints implied by its known hard X-ray variability on scales of days (Caballero-Garcia et al. 2012) and months (Fedorova et al. 2011). The MYTorus best fit parameters are reported in Table 3.

\subsubsection{IC 2560}

The barred spiral galaxy IC 2560 hosts a $(3.5 \pm 0.5) \times 10^{6} M_{\odot}$ active SMBH surrounded by a thin molecular maser disk (Yamauchi et al. 2012) with uncertain geometry (Tilak et al. 2008). The hard X-ray spectrum is well known from previous studies to be reflection-dominated (Baloković et al. 2014; Brightman et al. 2015). A default MYTorus model cannot reproduce a reflection-dominated spectrum $\left(\chi^{2} / v=364 / 107\right)$. A decoupled version of this model does better $\left(\chi^{2} / v=194 / 105\right)$, where all the radiation is back-scattered by Compton-thick material. The best fit is obtained with the Torus model $\left(\chi^{2} / v=\right.$ $172 / 107)$, and adding a line component at $(6.49 \pm 0.06) \mathrm{keV}$ significantly improves the fit $\left(\chi^{2} / v=127 / 105\right.$, Fig. 1h). The column density is found to be $>6.7 \times 10^{24} \mathrm{~cm}^{-2}$. Best fit parameters are given in Table 3 and agree with previous results in the literature.

\subsection{Summary of spectral analysis results}

In this section we presented hard X-ray spectral analyses for eight of the megamaser sources observed by NuSTAR. Three quarters turn out to be Compton-thick, while one quarter are Compton-thin. Among the latter, NGC 2960 is a $3 \sigma$ detection in the NuSTAR snapshot, while NGC 4388 is a well known variable source, presenting column density variability on the scale of days. 
Moreover, we note that out of seven sources showing a line feature (we exclude NGC 2960 in this argument, because of its weak detection), two do not have their line component well fit by self-consistent models (Torus or MYTorus). In both cases (NGC 1386 and IC 2560), the line was underestimated by the models. Finally, we note that using a decoupled MYTorus model in the Compton-thin regime (such as in the case of NGC 4388) should be done with caution, since the scattered components could mimic the transmitted primary continuum.

However, in this paper we are primarily interested in a robust estimate of the absorption column density, rather than an exhaustive discussion of the spectral properties of individual sources, which will be presented elsewhere (Baloković et al., in prep.). As previously stated, the final sample is completed by adding three NuSTAR-observed well known megamasers (NGC 4945, NGC 1068, Circinus), and three other maser disk AGN whose $\mathrm{X}$-ray (XMM-Newton based) and maser disk parameters are taken from Castangia et al. (2013). Summarizing, the fraction of Compton-thick AGN in our final sample of local disk megamasers is at least $\sim 79 \%(11 / 14)$, comparable to the values reported in previous studies $(76 \%$ - Greenhill et al. 2008 ; $86 \%$ Castangia et al. 2013) and confirming the tight relation between heavy obscuration and disk maser emission.

\section{The connection between the maser disk and the torus}

The aim of this paper is to deepen our understanding of the connection between the torus (seen as the X-ray obscurer) and the maser disk (i.e., an ensemble of clouds orbiting the central black hole, showing water maser activity). First, we can localize the disk. The maser emission occurs too far from the central black hole (in our sample, inner maser radii range from $6.6 \times 10^{4}$ to $7.6 \times 10^{6}$ gravitational radii) to identify the maser disk with the standard accretion disk, which extends up to $\sim 10^{3}$ gravitational radii (Netzer 2013, see Sect. 7.6, pp. 213-216). Moreover, the presence of water molecules requires the environment to be dusty. We then expect that the maser disk lies outside the dust sublimation radius $R_{\mathrm{d}}$, which identifies the torus inner wall within the standard AGN framework (see, e.g., Netzer 2015). We used the relation from Gandhi et al. (2009) to calculate $R_{\mathrm{d}}$ for our sample, adopting a sublimation temperature of $1500 \mathrm{~K}$ for graphite grains with an average radial size of $0.05 \mu \mathrm{m}$ (Barvainis 1987; Kishimoto et al. 2007). As expected, comparing $R_{\mathrm{d}}$ with the inner maser radius $R_{\text {in }}$ and considering the uncertainties, all the sources have the maser disk within the dusty zone (i.e., $R_{\text {in }} \geq$ $R_{\mathrm{d}}$ ), except NGC 2273 (as already pointed out by Castangia et al. 2013).

The maser disk can then be generally considered part of the torus with two different possible geometries: one in which the maser disk is the inner, sub-parsec scale part of the equatorial plane of the classical torus, as in Fig. 2a; and one in which the masing clouds are tracing a real geometrically thin disk that then inflates into a geometrically thicker end, required to have a large covering factor, as shown in Fig. 2b. There are many cases in which the maser disk is seen to be warped (NGC 2273, NGC 2960, Kuo et al. 2011; NGC 4258, Herrnstein et al. 2005; NGC 6264, Kuo et al. 2011; Circinus, Greenhill et al. 2003), or inflating in its outer part (NGC 3079, Kondratko et al. 2005). In particular, in the case of Circinus, the warp is consistent with channeling the nuclear outflow.

These geometries are difficult to explain within the framework of Fig. 2a. Moreover, the basic model of astrophysical

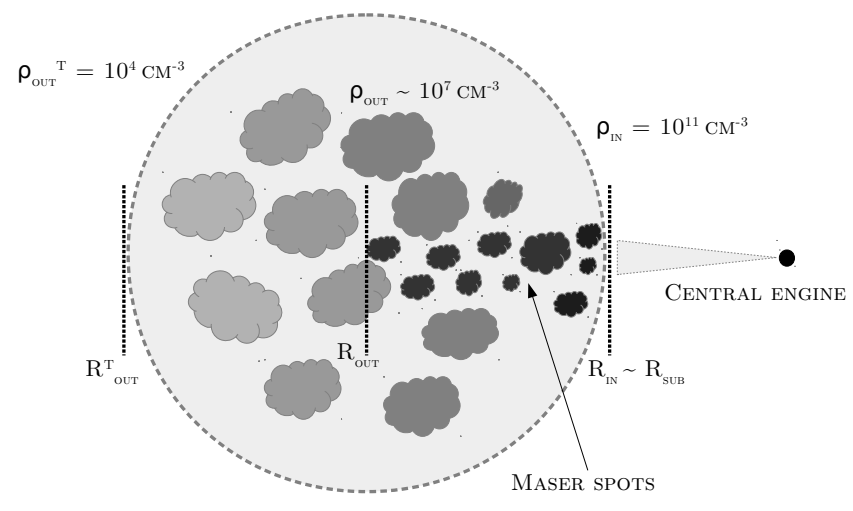

(a)

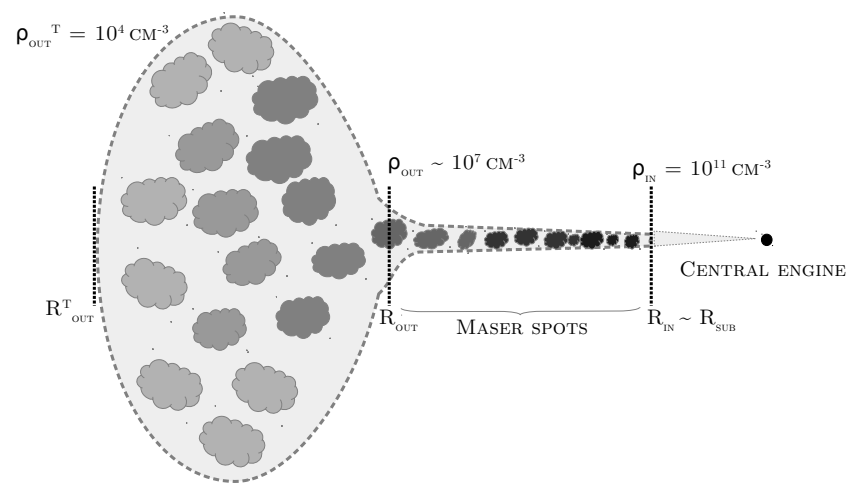

(b)

Fig. 2. Sketches of the two possible geometries for the location of the maser disk inside the torus. Sizes are not to scale, and the sketches are just meant to display the different possible locations of the maser disk. a) The disk is part of the equatorial plane of the torus. b) The disk inflates in its outer part, giving rise to a geometrically thicker structure. The change from the inner part to the outer one is not abrupt and occurs with a gradual change in the dimensions and physical conditions of the clumps, encoded in the same density profile.

maser emission theory predicts that the disk should be directly irradiated by X-rays coming from the central source (Neufeld et al. 1994). Again, this makes it difficult to explain the emerging disk when considering a geometry like Fig. 2a. Finally, a steep density gradient in the vertical direction would be needed to only see edge-on maser emission; otherwise, maser disks would be ubiquitous among Sy2 galaxies, contrary to observations (Zhu et al. 2011). Here we do not have information on the torus vertical structure, but we can exploit the physical properties of the maser emission to infer something about the most likely geometry. High densities, nearly edge-on geometry, and a temperature range of $\sim 400-1000 \mathrm{~K}$ are needed to have maser amplification (Lo 2005).

In this work, we concentrate only on the density condition. It is indeed difficult to estimate the temperature of the masing gas that is not, by definition, in thermodynamic equilibrium. Instead, we can estimate the density of the masing region, defining the maser disk radial extent $\Delta R=R_{\text {out }}-R_{\text {in }}$, where $R_{\text {out }}$ and $R_{\text {in }}$ are the outer and inner radii of the maser disk. They are taken as the locations of the less red/blueshifted and most red/blueshifted maser spots with respect to the systemic velocity of the galaxy, 
if using the spectrum, or the innermost and outermost spots whether from systemic or red/blueshifted masers, if using the maps. With the disk extent and the column density measured from the X-rays, $N_{\mathrm{H}}$, we have a rough estimate of the mean density of the material along the line of sight:

$\rho=\frac{N_{\mathrm{H}}}{\Delta R}\left[\mathrm{~cm}^{-3}\right]$

which we can compare with the densities predicted by astrophysical maser theory, $10^{7}<\rho<10^{11} \mathrm{~cm}^{-3}$ (Lo 2005; Tarchi 2012).

Looking at the density distribution in our sample, it is clear that densities obtained with (1) are too low by at least one order of magnitude. This is a hint that using (1) and identifying the absorbing medium with the maser disk is not completely appropriate, and if so, all megamaser sources in the sample should be severely obscured. Indeed, with an average density of $10^{9} \mathrm{~cm}^{-3}$ in a fraction of a parsec, the column density of such a maser disk should be on the order of $10^{26} \mathrm{~cm}^{-2}$. This is clearly not the case, because 3 out of 14 sources are Compton-thin (i.e., $N_{\mathrm{H}}<1.5 \times 10^{24} \mathrm{~cm}^{-2}$ ), and 6 out of 14 are Compton-thick with $N_{\mathrm{H}}<10^{25} \mathrm{~cm}^{-2}$.

In general, the maser disk alone cannot replace the standard torus of the AGN unified model: it is too geometrically thin (otherwise nearly every Sy2 would be identified as a maser source, while nuclear water maser emission detection frequency is low, $\sim 3 \%$, Zhu et al. 2011) and too optically thick. There could be cases, however, in which a warped disk could simultaneously provide enough obscuration and low covering factor. We discuss this possibility in Sect. 4.3. Indeed, five sources of the sample present a lower limit on the column density and are therefore consistent with the absorber being the maser disk itself, seen exactly edge-on. Because the density increases overall, approaching the black hole, we may simply guess that the maser spots are detected in a high-density region in the inner part of the torus. Moreover, we can explain the tight relation between high obscuration and edge-on maser emission as a co-alignment between the maser clouds and the obscuring matter. In what follows, we then assume geometric alignment and continuity in the radial density profile between the maser disk and the inflated part of the torus, adopting a geometry as in Fig. 2b.

An alternative view of the result obtained with Eq. (1) involves clumpiness, which is addressed quite well by theoretical models. Models like the one by Elitzur \& Shlosman (2006) study the interplay between the maser disk and the obscuring medium, followed by subsequent relevant work on this topic (e.g., Nenkova et al. 2008a,b). Dusty and molecular clouds orbiting the central engine are expected to have column densities in the range $N_{\mathrm{H}} \sim 10^{22}-10^{23} \mathrm{~cm}^{-2}$, and a few clouds are able to provide the necessary obscuration measured with X-ray spectroscopy, together with rapid variability and the radiation reprocessing in the infrared band. Even if many questions are still unanswered, these works point toward the importance of considering a clumpy medium, rather than a smooth one, to interpret and explain many properties of AGN. In this paper, we use analytical expressions of average quantities, like the density, to get our results. Later on, we test whether this methodology is too simplistic or not.

\subsection{A toy model}

Suppose now that the inner and outer radii of the maser disk correspond to the theoretically expected upper and lower limits

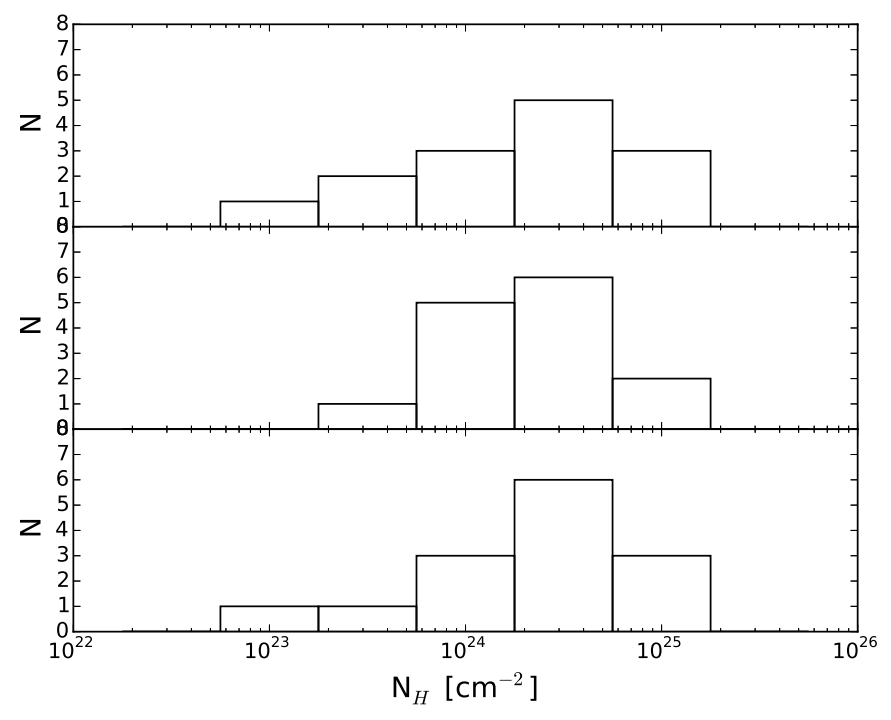

Fig. 3. Top panel: distribution of $N_{\mathrm{H}}$ as measured by X-ray spectral fitting. Middle panel: distribution of column densities predicted by the model, using a power law density profile. Bottom panel: distribution of column densities predicted by the model, with mixed exponential density profile (see text).

in density suitable to have maser emission, respectively, and assume a power law for the density profile, such as

$\rho(r)=\rho_{\text {in }}\left(\frac{r}{R_{\text {in }}}\right)^{-\alpha}$,

where $\alpha$ is the power law index, which can be estimated for every source taking $\rho_{\text {in }}=10^{11} \mathrm{~cm}^{-3}$ and $\rho_{\text {out }}=10^{7} \mathrm{~cm}^{-3}$ :

$\alpha=\log \left(\frac{\rho_{\text {in }}}{\rho_{\text {out }}}\right) / \log \left(\frac{R_{\text {out }}}{R_{\text {in }}}\right)=\frac{4}{\log \left(\frac{R_{\text {out }}}{R_{\text {in }}}\right)}$.

Once we have recovered the power law index for each source using the maser disk sizes from Table 1, a continuity assumption in the radial density profile between the maser disk and the external part of the torus allows us to estimate the torus outer radius. Identifying the outer end of the maser disk with the beginning of the inflated end and keeping the same $\alpha$, the torus outer radius $R_{\text {out }}^{\mathrm{T}}$ will be the distance at which the density falls to, say, $10^{4} \mathrm{~cm}^{-3}$ (see Netzer 2013, Sect. 7.5, p. 205). This value has a negligible effect on results, as we show in the following:

$R_{\text {out }}^{\mathrm{T}}=R_{\text {out }} 10^{\frac{1}{\alpha} \log \left(\rho_{\text {out }} / \rho_{\text {out }}^{\mathrm{T}}\right)}=R_{\text {out }} 10^{3 / \alpha}$.

Assuming a density profile continuity between the maser disk and the external part of the torus, with this simple toy model one can recover the torus size, and then integrate its density along the line of sight inside the inflated part alone, to recover the column density:

$N_{\mathrm{H}}=\int_{R_{\text {out }}}^{R_{\text {out }}^{\mathrm{T}}} \rho(r) \mathrm{d} r=\frac{\rho_{\text {out }} R_{\text {out }}}{\alpha-1}\left[1-\left(\frac{R_{\text {out }}^{\mathrm{T}}}{R_{\text {out }}}\right)^{1-\alpha}\right]$.

Very interestingly, column densities calculated with (5) are in good agreement with the ones measured with X-ray spectral fitting (Fig. 3, middle panel). Moreover, it turns out that between the tunable parameters of the model (the densities $\rho_{\text {in }}$ and $\rho_{\text {out }}$ at which the maser disk begins and ends, and $\rho_{\text {out }}^{\mathrm{T}}$ at which the torus ends), results are only sensitive to the outer maser disk density, $\rho_{\text {out }}$. This can be seen directly from Eq. (5). In particular, 


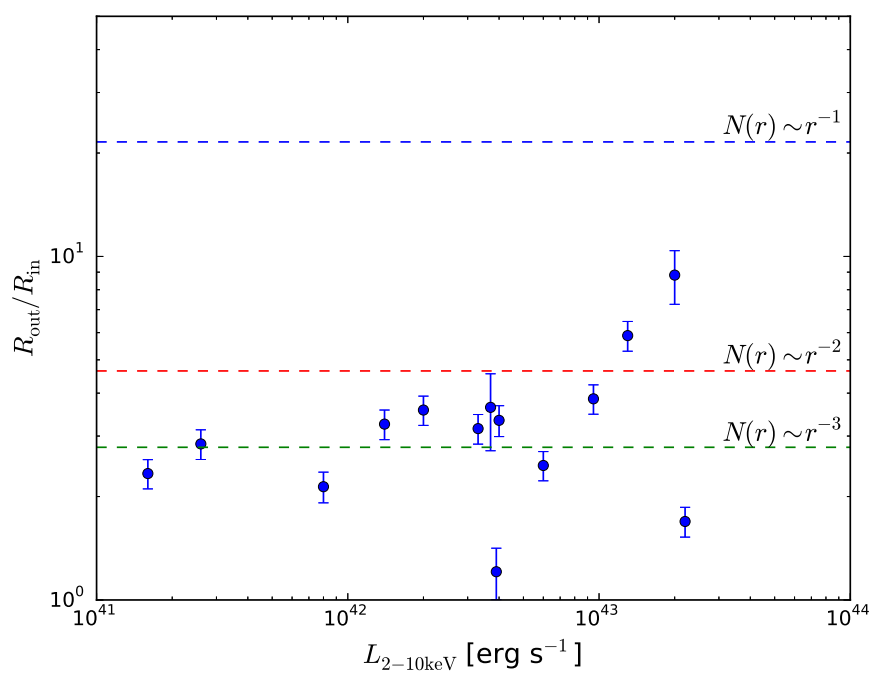

Fig. 4. Ratio of outer and inner maser radii for the sources in the sample as a function of X-ray (deabsorbed) luminosity in the $2-10 \mathrm{keV}$ band. The trend $N(r) \sim r^{-1}$ (blue dashed line) seems to be ruled out, while $N(r) \sim r^{-2}$ and $N(r) \sim r^{-3}$ (red and green dashed lines, respectively) are preferred. We note that the ratios expected for different clouds distributions depend on the critical maser densities assumed, being the expected ratio for a particular $q, R_{\text {out }} / R_{\text {in }}=10^{\log \left(\rho_{\text {in }} / \rho_{\text {out }}\right) / 3 q}$.

changing $\rho_{\text {out }}$ by one order of magnitude changes $N_{\mathrm{H}}$ by a factor $\sim 14$, while the same variation in $\rho_{\text {in }}$ and $\rho_{\text {out }}^{\mathrm{T}}$ has a negligible impact on the distribution (factor $\sim 1.3$ and $\sim 1.01$, respectively). In other words, a small change in the parameter $\rho_{\text {out }}$ gives a large change in the recovered $N_{\mathrm{H}}$, and this is a hint that the theoretically driven choice of the three densities is the best at reproducing the observed column density distribution.

Another step forward can be made by testing the power law assumption for the density profile. The $\alpha$ parameter in fact only tells how fast the density falls inside the maser disk, decreasing by $\sim 4$ orders of magnitude in a fraction of a parsec. The resulting distribution of the $\alpha$ parameter is skewed and very steep, peaking at very high values $(\sim 8)$.

Clouds orbiting a SMBH at sub-parsec distances are often modeled with a radial dependence of the form $N(r) \sim r^{-q}$, where $N(r)$ is the number of clouds per unit length, and $q$ is usually 1 or 2 (e.g., Nenkova et al. 2008b). This translates in a radial dependence of the number of clouds per unit volume of $\sim r^{-3 q}$. If every cloud has approximately the same number of atoms and same chemical composition, the same radial trend holds also for the density $\rho$ to which we refer here. In Fig. 4 we show how our $\alpha$ indexes, which describe the density falling rate in a smooth medium, compare with the power law distributions of clouds in clumpy models. In Fig. 4 we plot the ratio of the outer and inner maser radii as a function of X-ray (deabsorbed) luminosity in the $2-10 \mathrm{keV}$ band. There is no clear correlation between the two quantities. The ratios cluster roughly between the values 2-4. We also plot the ratios expected for different radial distributions with dashed lines of different colors, showing that $q$ indexes of 2-3 are preferred. One possibility for explaining this trend is that the density gradient between the inner and outer maser radii is lower than the assumed one (four orders of magnitude, from $10^{11} \mathrm{~cm}^{-3}$ at $R_{\text {in }}$ to $10^{7} \mathrm{~cm}^{-3}$ at $\left.R_{\text {out }}\right)$. For example, a decrease of three orders of magnitude (which is reasonable, assuming current uncertainties) would make the data fully consistent with a distribution of clouds with power law index $q=2$. Another possibility is that in a real medium, which is probably a mixture of clumps, voids, and filaments, the density falls abruptly between clouds, steepening the $\alpha$ index. Also warps in maser disks could bias the inner and outer maser radii measurements. Taking the caveats and uncertainties described above into account, we conclude that our analytical formulas for the density are consistent with a radial distribution of clouds $N(r) \sim r^{-q}$, with $q \sim 2,3$.

An alternative modelization of our steep density profile is an exponential one:

$\rho(r)=\rho_{\text {in }} \exp \left[-\left(\frac{r-R_{\text {in }}}{R_{\text {out }}-R_{\text {in }}}\right)^{1 / n} \ln \left(\frac{\rho_{\text {in }}}{\rho_{\text {out }}}\right)\right]$,

where $n$ is the equivalent of the Sersic index. We note that the $n=1$ and $n=2$ cases are, in general, able to represent Comptonthin and Compton-thick sources, respectively. In other words, results similar to the power law case are found for a mixed density profile, different between Compton-thin and thick sources.

For each $n$, the torus outer radius and the column density can be calculated with

$$
\begin{aligned}
R_{\text {out }}^{\mathrm{T}} & =R_{\text {in }}+\left(R_{\text {out }}-R_{\text {in }}\right)\left[\frac{\ln \left(\rho_{\text {out }}^{\mathrm{T}} / \rho_{\text {in }}\right)}{\ln \left(\rho_{\text {out }} / \rho_{\text {in }}\right)}\right]^{n} \\
N_{\mathrm{H}} & =\frac{n\left(R_{\text {out }}-R_{\text {in }}\right)}{\left[\ln \left(\rho_{\text {in }} / \rho_{\text {out }}\right)\right]^{n}} \rho_{\text {in }}\left[\Gamma\left(n, \ln \frac{\rho_{\text {in }}}{\rho_{\text {out }}}\right)-\Gamma\left(n, \ln \frac{\rho_{\text {in }}}{\rho_{\text {out }}^{\mathrm{T}}}\right)\right],
\end{aligned}
$$

where $\Gamma(n, x)$ is the incomplete Gamma function, and $\Gamma(n, 0)=$ $\Gamma(n)$. In the specific cases $n=1$ and $n=2$, Eq. (8) becomes

$$
N_{\mathrm{H}}=\left\{\begin{array}{ll}
\frac{\left(R_{\text {out }}-R_{\text {in }}\right)}{\ln \left(\rho_{\text {in }} / \rho_{\text {out }}\right)}\left(\rho_{\text {out }}-\rho_{\text {out }}^{\mathrm{T}}\right) & n=1 \\
\frac{2\left(R_{\text {out }}-R_{\text {in }}\right)}{\left[\ln \left(\rho_{\text {in }} / \rho_{\text {out }}\right)^{2}\right.}\left\{\rho_{\text {out }}\left[\ln \left(\frac{\rho_{\text {in }}}{\rho_{\text {out }}}\right)+1\right]-\rho_{\text {out }}^{\mathrm{T}}\left[\ln \left(\frac{\rho_{\text {in }}}{\rho_{\text {out }}^{\mathrm{T}}}\right)+1\right]\right\} & n=2
\end{array} .\right.
$$

We can then repeat the same analysis, using Eqs. (7) and (9) to predict the column density distribution with fixed $\rho_{\text {out }}=$ $10^{7} \mathrm{~cm}^{-3}$ (Fig. 3, bottom panel): notably, the mixed exponential density profile (i.e. with $1 \leq n \leq 2$ ) can reproduce the observed distribution of X-ray measured column densities better than the power law general case.

\subsection{The torus size}

Instead of assuming the transition density $\rho_{\text {out }}$ to infer $N_{\mathrm{H}}$, we now use the column densities measured by NuSTAR and reverse the problem. Inverting (9), one can calculate the parameter $\rho_{\text {out }}$ needed to have a torus with a column density equal to the measured one. The result is that it is sufficient to have a sharp distribution of $\rho_{\text {out }}$ peaked at $10^{7} \mathrm{~cm}^{-3}$ to have tori with the measured column densities. In other words, fixing the inner maser density $\rho_{\text {in }}$ and the outer torus density $\rho_{\text {out }}^{\mathrm{T}}$ and using the measured column densities, the model points toward a transition density of about $10^{7} \mathrm{~cm}^{-2}$, without knowing anything of the previous theoretical assumptions.

Figure 5 shows the resulting $\rho_{\text {out }}$ distribution (see Table 4 for numerical values), while Fig. 6 shows the torus outer radius distribution obtained using Eq. (7) (numerical values are reported in Table 4).

\section{Discussion}

\subsection{Comparison with mid-infrared interferometry}

Our toy model allows us to predict the X-ray column density distribution of a sample of disk maser systems or to calculate the 


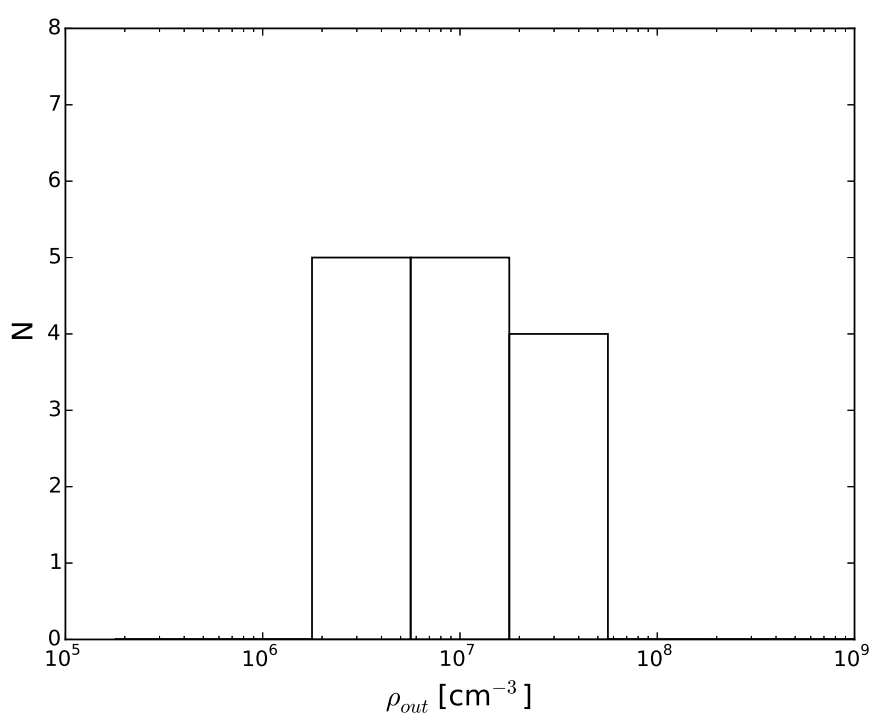

Fig. 5. Distribution of the parameter $\rho_{\text {out }}$ in the sample, calculated as described in Sect. 3.2. See also Table 4.

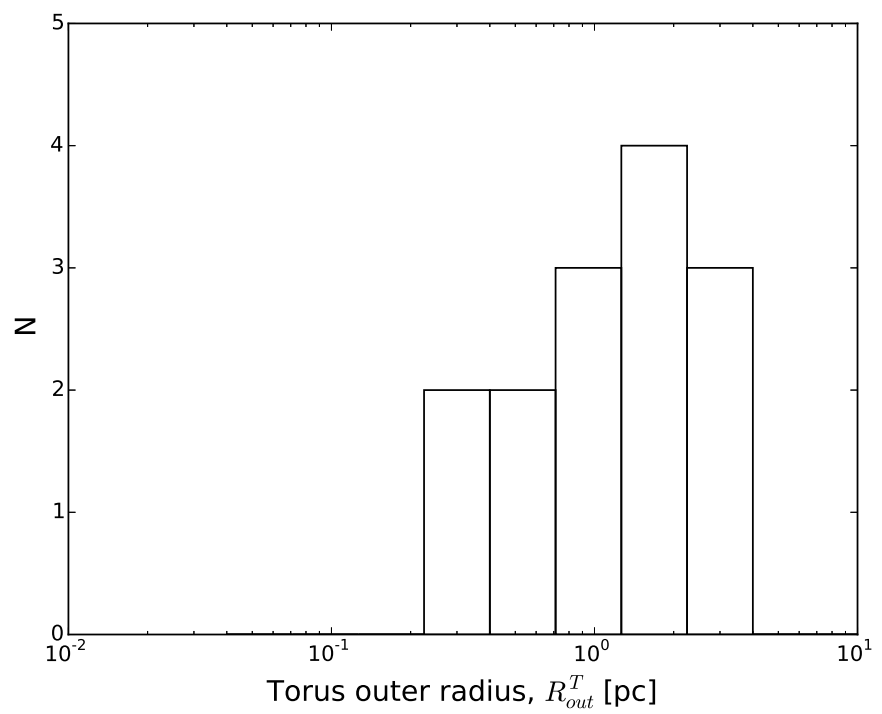

Fig. 6. Distribution of torus outer radius predicted by the model, calculated as described in Sect. 3.2. See also Table 4.

torus outer radius if the column density is known. When dealing with very high column densities (i.e., lower limits on $N_{\mathrm{H}}$ ), the torus outer radius is poorly constrained, since the measured column density can be ascribed to the maser disk without the need of an inflated torus. However, it is interesting to compare our results with mid-infrared (MIR) measurements, which are thought to probe the dusty structure surrounding AGN. In our sample, only NGC 1068 (Raban et al. 2009; López-Gonzaga et al. 2014) and Circinus (Tristram et al. 2007, 2014) have been observed with MIR interferometry. In both sources two distinct structures responsible for the MIR emission are detected. One is an elongated, disk-like structure, which is co-aligned and cospatial with the maser emission spots and perpendicular to the ionization cones (albeit with the caveat of uncertainty in the absolute astrometry). The second structure, whose origin and theoretical explanation is still unclear, seems to be responsible for diffuse emission on much larger scales $(>1 \mathrm{pc})$, broadly perpendicular to the first. The geometry of this double dusty structure is currently challenging the classical torus framework. These
Table 4. $\rho_{\text {out }}$ values needed to have $N_{\mathrm{H} \text {,meas }}=N_{\mathrm{H} \text {,pred }}$ with the assumption of maser disk-torus density profile continuity, and output of the model, the torus outer radius.

\begin{tabular}{lrr}
\hline \hline Name & $\rho_{\text {out }}\left[\mathrm{cm}^{-3}\right]$ & $R_{\text {out }}^{\mathrm{T}}[\mathrm{pc}]$ \\
\hline NGC 1194 & $2.8 \times 10^{6}$ & $2.4 \pm 0.2$ \\
NGC 1386 & $1.3 \times 10^{7}$ & $2.1 \pm 0.3$ \\
NGC 2273 & $4.8 \times 10^{7}$ & $0.77_{-0.07}^{+\infty}$ \\
NGC 2960 & $6.5 \times 10^{6}$ & $0.53_{-0.05}^{+0.06}$ \\
NGC 3079 & $4.1 \times 10^{6}$ & $2.7 \pm 0.2$ \\
NGC 3393 & $2.6 \times 10^{6}$ & $3.3 \pm 0.5$ \\
NGC 4388 & $2.4 \times 10^{7}$ & $0.34 \pm 0.07$ \\
IC 2560 & $3.1 \times 10^{7}$ & $1.1_{-0.1}^{+\infty}$ \\
NGC 1068 & $1.6 \times 10^{7}$ & $2.2_{-0.3}^{+\infty}$ \\
NGC 4945 & $1.6 \times 10^{7}$ & $1.1 \pm 0.1$ \\
Circinus & $3.4 \times 10^{7}$ & $1.3 \pm 0.3$ \\
NGC 4258 & $1.9 \times 10^{6}$ & $0.36 \pm 0.03$ \\
NGC 6264 & $2.8 \times 10^{6}$ & $1.6_{-0.2}^{+\infty}$ \\
UGC 3789 & $6.6 \times 10^{6}$ & $0.69_{-0.06}^{+\infty}$ \\
\hline
\end{tabular}

Notes. Six of our sources have a lower limit on the column density and therefore on the torus outer radius, too. In these cases, the derived torus outer radius may be less constrained owing to the high obscuring column, compatible with the maser disk one.

two-component structures are not considered in our simple toy model; however, we note that the sizes are in broad agreement with our predictions, being parsec scales. Another possibility of comparison comes from considering half-light radii $\left(r_{1 / 2}\right)$, enclosing half of the MIR flux of the source, as done in Burtscher et al. (2013). We note that the NGC 1068 and Circinus outer radii are less than a factor of two larger than the $r_{1 / 2}$ values reported by Burtscher et al. (2013). This could be expected, since the outer torus radius should be larger than the half-light one. Moreover, the $r_{1 / 2}$ of NGC 1068 is broadly twice the $r_{1 / 2}$ of Circinus; the same happens with $R_{\text {out }}^{\mathrm{T}}$ in our toy model. Future observations in the MIR band of other sources are needed to probe this scenario.

\subsection{Trend with bolometric luminosity}

Burtscher et al. (2013) found a clear positive trend of the halflight radius (used as a proxy for the torus size) with the bolometric luminosity, although with large scatter (their Fig. 36). In the near-infrared (NIR), a scaling of the dust sublimation radius with $L_{\text {bol }}^{1 / 2}$ is well known. However, this relation is much more scattered in the MIR: more luminous sources generally have larger tori with no clear trend. We can then explore whether a relation between the bolometric luminosity and the torus size holds in our toy model. We take $L_{\text {bol }}=\kappa_{\text {bol }} \times L_{2-10}^{\text {int }}$, where $\kappa_{\text {bol }}=20 \pm 5$ is the bolometric correction, which is constant in our range of intrinsic 2-10 keV luminosities (Steffen et al. 2006; Lusso et al. 2012). We choose a $25 \%$ uncertainty on $\kappa_{\text {bol }}$ to also include a reasonable error on the $2-10 \mathrm{keV}$ intrinsic luminosity derived from NuSTAR spectral fitting, and we note that using a non-constant bolometric correction would steepen the correlations. We fit our sample with a linear relation of the form $\log y=a\left(\log L_{\mathrm{bol}}-43.5\right)+b \pm S$, where $L_{\mathrm{bol}}$ is measured in $\mathrm{erg} \mathrm{s}^{-1}$ and $S$ represents the intrinsic scatter in the relation. We applied a Bayesian analysis with loose priors (uniform for all the unknown parameters, i.e., the slope, the intercept, and the intrinsic scatter). Since our tori are an extension of the maser disks, we first explored the possibility of a correlation between the inner and 
Table 5. $R_{\mathrm{in}}-L_{\mathrm{bol}}, R_{\mathrm{out}}-L_{\mathrm{bol}}$, and $R_{\mathrm{out}}^{\mathrm{T}}-L_{\mathrm{bol}}$ relations: best fit parameters.

\begin{tabular}{lccc}
\hline \hline Parameter & $R_{\text {in }}-L_{\text {bol }}$ & $R_{\text {out }}-L_{\text {bol }}$ & $R_{\text {out }}^{\mathrm{T}}-L_{\text {bol }}$ \\
\hline$a$ & $0.02_{-0.17}^{+0.16}$ & $0.12_{-0.14}^{+0.14}$ & $0.30_{-0.13}^{+0.13}$ \\
$b\left(\right.$ at $\left.\log L_{\text {bol }} / \mathrm{erg} \mathrm{s}^{-1}=43.5\right)$ & $-0.75_{-0.12}^{+0.12}$ & $-0.29_{-0.10}^{+0.09}$ & $0.04_{-0.09}^{+0.09}$ \\
$S$ [dex $]$ & $0.42_{-0.09}^{+0.09}$ & $0.34_{-0.07}^{+0.07}$ & $0.30_{-0.07}^{+0.07}$ \\
\hline
\end{tabular}

Notes. Errors quoted are $1 \sigma$ confidence level.

outer maser radii with bolometric luminosity. Results are shown in Figs. 7a and b. Finally, we repeated the same procedure for the torus outer radius, which is a derived quantity (Fig. 7c-refer to Table 5 for best fit parameters in all three cases). We find an interesting evolution of the trend, going from the absence of a correlation between the inner maser radius and the bolometric luminosity to a positive correlation between the torus outer radius and luminosity, although with large intrinsic scatter. The slopes are, however, all consistent within the uncertainties.

The weak trend of the torus's outer radius with luminosity could also reflect the weak correlation between maser disks dimensions with luminosity already noted by Greenhill et al. (2003) comparing NGC 4258 and Circinus inner maser radii. We have here confirmed that finding with an enlarged sample. As already suggested by Greenhill et al. (2003), warps in maser disks could break the edge-on geometry condition and bias the disk radial extent measurements.

\subsection{The possible role of warps}

Warped disks have been observed in some sources of the sample (Circinus and NGC 4258 are the clearest cases, see Greenhill et al. 2003; Wu et al. 2013, and references therein). Low covering factor and/or fast $N_{\mathrm{H}}$ variability (as in the case of NGC 4945, see, e.g., Madejski et al. 2000; Puccetti et al. 2014) could indicate the maser disk as the obscuring structure, instead of invoking an inflated torus. It is easy to see that, when keeping the densities expected by the astrophysical maser theory, only a small fraction of the disk is required to intercept the line of sight to have the measured column density (see Fig. 8). To calculate the radial extent of such a warp, we define $R_{\mathrm{w}}$ as the warping radius and assume that the warp extends up to the maser outer radius, $R_{\text {out }}$. To calculate $R_{\mathrm{w}}$, it is sufficient to replace $\rho_{\text {out }}$ with $\rho_{\mathrm{w}}$ and $\rho_{\text {out }}^{\mathrm{T}}$ with $\rho_{\text {out }}$ in Eqs. (7) and (9), if adopting an exponential density profile. If using a power law density profile, results are the same within the uncertainties.

In a picture in which there is no standard torus, but only a nearly edge-on molecular disk, a warp of depth $\Delta R_{\mathrm{w}}=R_{\text {out }}-R_{\mathrm{w}}$ is required to obscure the central engine. Numerical values can be found in Table 6 .

\section{Conclusions}

We presented hard X-ray spectral analyses of NuSTAR data for eight sources out of a sample of 14 nearby disk megamaser galaxies with the aim of exploring the relationship between the maser disk and the environment in which it resides. In our final sample of $14 \mathrm{AGN}, 79 \%$ are Compton-thick, and $21 \%$ are Compton-thin. All these objects are indeed obscured Sy2 galaxies, and show $22 \mathrm{GHz}$ maser emission from water vapor molecules in a dense molecular disk around active SMBHs. We proposed a toy model to explain this connection, where the

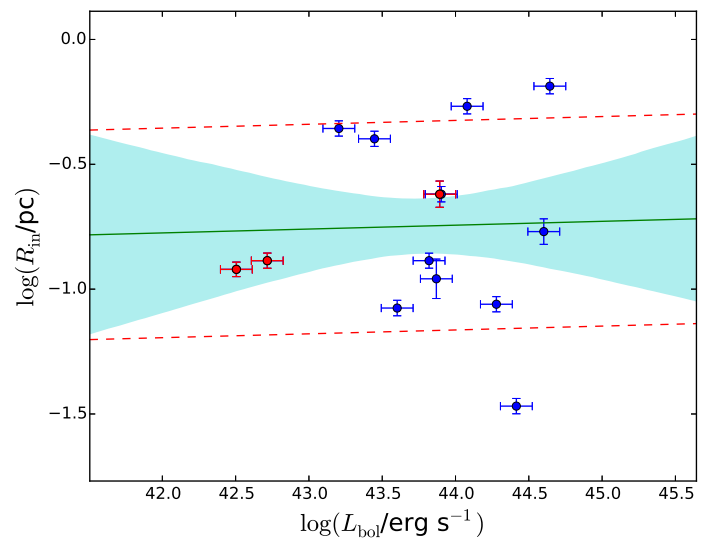

(a)

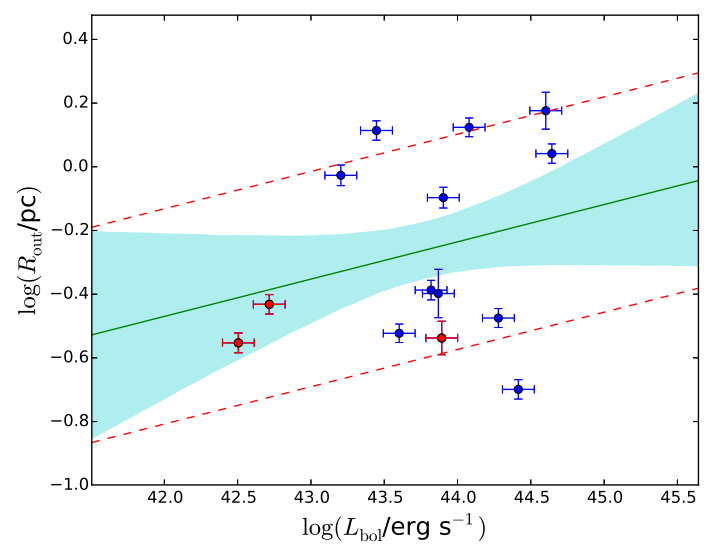

(b)

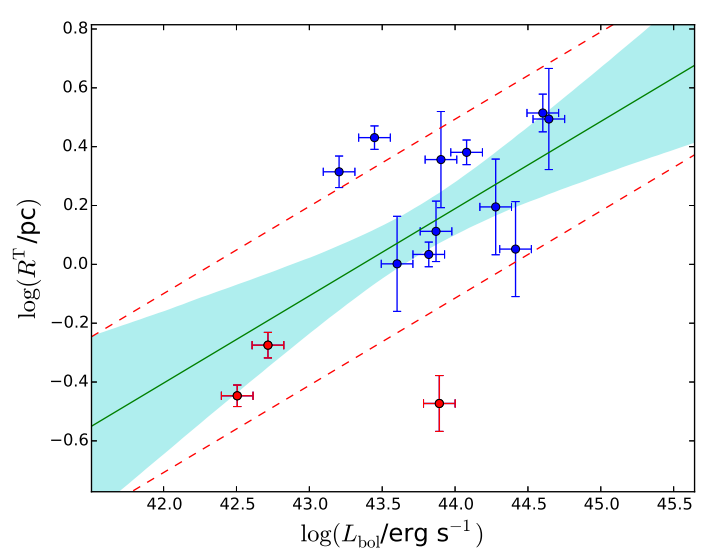

(c)

Fig. 7. Size-luminosity relations, with progression from an absence of correlation (7a) to a possible one (7c). a) Maser disk inner radius as a function of bolometric luminosity, $L_{\mathrm{bol}}$. The three Compton-thin sources are indicated by red points. The green line is the best fit linear model, the red dashed lines show the intrinsic scatter of the data, and the cyan shaded area indicates the uncertainty of the model. b) Maser disk outer radius as a function of bolometric luminosity, $L_{\mathrm{bol}}$. Colors and symbols are the same as in the upper panel. c) Predicted torus outer radius as a function of bolometric luminosity, $L_{\mathrm{bol}}$. Colors and symbols are the same as in the upper panels.

maser disk is the inner part of the torus, ending in an inflated, geometrically thicker structure. Even if the model is simplistic, it is able to recover the column density distribution for a sample of obscured, disk megamaser AGN, using reasonable density 
Table 6. $\Delta R_{\mathrm{W}}$ values needed to have $N_{\mathrm{H} \text {,meas }}=N_{\mathrm{H} \text {,pred }}$ with the assumption of all obscuration due to a warp of the maser disk entering the line of sight.

\begin{tabular}{lrr}
\hline \hline Name & $R_{\mathrm{w}}[\mathrm{pc}]$ & $\Delta R_{\mathrm{w}}[\mathrm{pc}]$ \\
\hline NGC 1194 & 1.29 & 0.04 \\
NGC 1386 & 0.84 & 0.09 \\
NGC 2273 & $<0.13$ & $>0.07$ \\
NGC 2960 & 0.36 & 0.01 \\
NGC 3079 & 1.23 & 0.07 \\
NGC 3393 & 1.44 & 0.06 \\
NGC 4388 & 0.283 & 0.007 \\
IC 2560 & $<0.25$ & $>0.08$ \\
NGC 1068 & $<1.0$ & $>0.1$ \\
NGC 4945 & 0.35 & 0.06 \\
Circinus & 0.3 & 0.1 \\
NGC 4258 & 0.277 & 0.003 \\
NGC 6264 & $<0.77$ & $>0.03$ \\
UGC 3789 & $<0.28$ & $>0.02$ \\
\hline
\end{tabular}

Notes. Uncertainties are neglected for clarity. Warp depths are lower than, or comparable to, the uncertainties on outer maser radii. The five sources with a lower limit on the column density consequently have a lower limit on the warp depth.

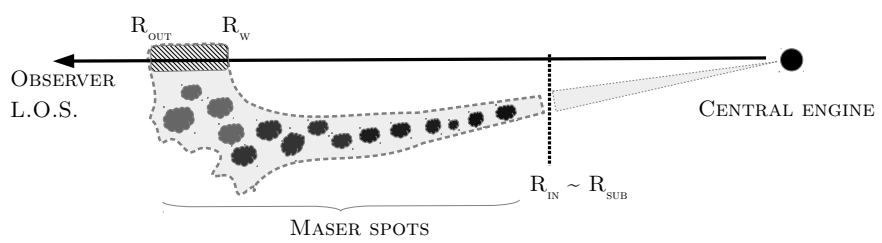

Fig. 8. Possible role of warps: rapid $N_{\mathrm{H}}$ variability and low covering factor. The obscuring column is provided by a warp of depth $\Delta R_{\mathrm{w}}=$ $R_{\text {out }}-R_{\mathrm{w}}$. The thick arrow denotes the observer line of sight.

profiles (a power law or, better, an exponential with $1 \leq n \leq 2$ ). Alternatively, one can start from the measured $R_{\text {in }}, R_{\text {out }}$, and $N_{\mathrm{H}}$, assume a reasonable density profile, solve the equations for the crucial parameter $\rho_{\text {out }}$, and estimate the torus outer radius, which is found to be on the parsec scale. A direct and robust measure of the torus size is available in two sources (NGC 1068 and Circinus) through mid-IR interferometry. In both cases, the outcomes of the model agree with the half-light radius or single resolved structure size measurements. Clearly, a more physical picture explicitly addressing the known disk/torus clumpiness and warping must rely on numerical calculations.

When we assume a geometry like the one proposed in Fig. 2b, the column density derived with X-ray spectroscopy is due to the inflated end of the clumpy torus alone. Indeed, in ten sources of our sample, $N_{\mathrm{H}}<10^{25} \mathrm{~cm}^{-2}$. This obscuration can be explained if the line of sight does not intercept the masing disk. In the remaining five sources, the column density is compatible with that of the maser disk, which is very optically thick in this framework: these sources could be those seen exactly edge-on, with the line of sight intercepting the geometrically thin maser disk along the equatorial plane. We note, however, that NGC 6264 and UGC 3789 currently lack NuSTAR spectra, and their column density has been estimated using just XMM-Newton (Castangia et al. 2013). Future NuSTAR observations may shed light on the exact value of the column density of these two sources, further constraining the fraction of heavily $\left(N_{\mathrm{H}}>10^{25} \mathrm{~cm}^{-2}\right)$ Compton-thick AGN in our sample. We finally discussed the possibility that, in some cases, warps in the maser disk may play the role of the classical torus in the AGN unified model, providing low covering factor and fast column density variability.

Acknowledgements. We thank the anonymous referee for useful suggestions that helped to improve the paper. This work was supported under NASA Contract NNG08FD60C, and it made use of data from the NuSTAR mission, a project led by the California Institute of Technology, managed by the Jet Propulsion Laboratory, and funded by the National Aeronautics and Space Administration. We thank the NuSTAR Operations, Software, and Calibration teams for support with the execution and analysis of these observations. This research made use of the NuSTAR Data Analysis Software (NuSTARDAS) jointly developed by the ASI Science Data Center (ASDC, Italy) and the California Institute of Technology (USA). A.M., A.C., and S.P. acknowledge support from the ASI/INAF grant I/037/12/0-011/13. M.B. acknowledges support from NASA Headquarters under the NASA Earth and Space Science Fellowship Program, grant NNX14AQ07H. We acknowledge support from CONICYT-Chile grants Basal-CATA PFB-06/2007 (F.E.B., C.R.), FONDECYT 1141218 (F.E.B., C.R.), "EMBIGGEN" Anillo ACT1101 (F.E.B., C.R.), and the Ministry of Economy, Development, and Tourism's Millennium Science Initiative through grant IC120009, awarded to The Millennium Institute of Astrophysics, MAS (F.E.B.). W. N.B. acknowledges support from NuSTAR subcontract 44A1092750 .

\section{References}

Aird, J., Alexander, D. M., Ballantyne, D. R., et al. 2015, ApJ, 815, 66 Antonucci, R. 1993, ARA\&A, 31, 473

Arévalo, P., Bauer, F. E., Puccetti, S., et al. 2014, ApJ, 791, 81

Arnaud, K. A. 1996, in Astronomical Data Analysis Software and Systems V, eds. G. H. Jacoby, \& J. Barnes, ASP Conf. Ser., 101, 17

Awaki, H., Terashima, Y., Higaki, Y., \& Fukazawa, Y. 2009, PASJ, 61, 317

Baloković, M., Comastri, A., Harrison, F. A., et al. 2014, ApJ, 794, 111

Barvainis, R. 1987, ApJ, 320, 537

Bauer, F. E., Arevalo, P., Walton, D. J., et al. 2015, ApJ, 812, 116

Braatz, J., Greenhill, L., Moran, J., Wilson, A., \& Herrnstein, J. 1997, in Am. Astron. Soc. Meet. Abstr., BAAS, 29, 104.02

Brightman, M., \& Nandra, K. 2011, MNRAS, 413, 1206

Brightman, M., Baloković, M., Stern, D., et al. 2015, ApJ, 805, 41

Burtscher, L., Meisenheimer, K., Tristram, K. R. W., et al. 2013, A\&A, 558, A149

Caballero-Garcia, M. D., Papadakis, I. E., Nicastro, F., \& Ajello, M. 2012, A\&A, 537, A 87

Cash, W. 1979, ApJ, 228, 939

Castangia, P., Panessa, F., Henkel, C., Kadler, M., \& Tarchi, A. 2013, MNRAS, 436, 3388

Churchwell, E., Witzel, A., Huchtmeier, W., et al. 1977, A\&A, 54, 969

Civano, F., Hickox, R. C., Puccetti, S., et al. 2015, ApJ, 808, 185

Comastri, A. 2004, in Multiwavelength AGN Surveys, eds. R. Mújica, \& R. Maiolino, 323

Elitzur, M., \& Shlosman, I. 2006, ApJ, 648, L101

Fedorova, E. V., Beckmann, V., Neronov, A., \& Soldi, S. 2011, MNRAS, 417, 1140

Gandhi, P., Horst, H., Smette, A., et al. 2009, A\&A, 502, 457

Gilli, R., Comastri, A., \& Hasinger, G. 2007, A\&A, 463, 79

Greenhill, L. J., \& Gwinn, C. R. 1997, Ap\&SS, 248, 261

Greenhill, L. J., Moran, J. M., \& Herrnstein, J. R. 1997, ApJ, 481, L23

Greenhill, L. J., Booth, R. S., Ellingsen, S. P., et al. 2003, ApJ, 590, 162

Greenhill, L. J., Tilak, A., \& Madejski, G. 2008, ApJ, 686, L13

Guainazzi, M., Fabian, A. C., Iwasawa, K., Matt, G., \& Fiore, F. 2005, MNRAS, 356,295

Harrison, F. A., Craig, W. W., Christensen, F. E., et al. 2013, ApJ, 770, 103

Harrison, F. A., Aird, J., Civano, F., et al. 2015, ApJ, submitted [arXiv: 1511.04183]

Henkel, C., Braatz, J. A., Reid, M. J., et al. 2012, in IAU Symp. 287, eds. R. S. Booth, W. H. T. Vlemmings, \& E. M. L. Humphreys, 301

Herrnstein, J. R., Moran, J. M., Greenhill, L. J., \& Trotter, A. S. 2005, ApJ, 629, 719

Kishimoto, M., Hönig, S. F., Beckert, T., \& Weigelt, G. 2007, A\&A, 476, 713

Kondratko, P. T., Greenhill, L. J., \& Moran, J. M. 2005, ApJ, 618, 618

Kondratko, P. T., Greenhill, L. J., \& Moran, J. M. 2008, ApJ, 678, 87

Koss, M. J., Romero-Canizales, C., Baronchelli, L., et al. 2015, ApJ, 807, 149

Kuo, C. Y., Braatz, J. A., Condon, J. J., et al. 2011, ApJ, 727, 20

Lo, K. Y. 2005, ARA\&A, 43, 625 
Lodato, G., \& Bertin, G. 2003, A\&A, 398, 517

López-Gonzaga, N., Jaffe, W., Burtscher, L., Tristram, K. R. W., \& Meisenheimer, K. 2014, A\&A, 565, A71

Lusso, E., Comastri, A., Simmons, B. D., et al. 2012, MNRAS, 425, 623

Madejski, G., Życki, P., Done, C., et al. 2000, ApJ, 535, L87

Magdziarz, P., \& Zdziarski, A. A. 1995, MNRAS, 273, 837

McConnell, N. J., \& Ma, C.-P. 2013, ApJ, 764, 184

Mullaney, J. R., Del-Moro, A., Aird, J., et al. 2015, ApJ, 808, 184

Murphy, K. D., \& Yaqoob, T. 2009, MNRAS, 397, 1549

Nenkova, M., Sirocky, M. M., Ivezić, Ž., \& Elitzur, M. 2008a, ApJ, 685, 147

Nenkova, M., Sirocky, M. M., Nikutta, R., Ivezić, Ž., \& Elitzur, M. 2008b, ApJ, 685,160

Netzer, H. 2013, The Physics and Evolution of Active Galactic Nuclei (Cambridge, UK: Cambridge University Press)

Netzer, H. 2015, ARA\&A, 53, 365

Neufeld, D. A., Maloney, P. R., \& Conger, S. 1994, ApJ, 436, L127

Pesce, D. W., Braatz, J. A., Condon, J. J., et al. 2015, ApJ, 810, 65

Puccetti, S., Comastri, A., Fiore, F., et al. 2014, ApJ, 793, 26

Raban, D., Jaffe, W., Röttgering, H., Meisenheimer, K., \& Tristram, K. R. W. 2009, MNRAS, 394, 1325
Reid, M. J., Braatz, J. A., Condon, J. J., et al. 2009, ApJ, 695, 287 Reid, M. J., Braatz, J. A., Condon, J. J., et al. 2013, ApJ, 767, 154 Risaliti, G., Maiolino, R., \& Salvati, M. 1999, ApJ, 522, 157

Steffen, A. T., Strateva, I., Brandt, W. N., et al. 2006, AJ, 131, 2826

Tarchi, A. 2012, in IAU Symp. 287, eds. R. S. Booth, W. H. T. Vlemmings, \& E. M. L. Humphreys, 323

Tilak, A., Greenhill, L. J., Done, C., \& Madejski, G. 2008, ApJ, 678, 701

Treister, E., Urry, C. M., \& Virani, S. 2009, ApJ, 696, 110

Tristram, K. R. W., Meisenheimer, K., Jaffe, W., et al. 2007, A\&A, 474, 837

Tristram, K. R. W., Burtscher, L., Jaffe, W., et al. 2014, A\&A, 563, A82

Urry, C. M., \& Padovani, P. 1995, PASP, 107, 803

Vasylenko, A. A., Fedorova, E. V., Hnatyk, B. I., \& Zhdanov, V. I. 2015, Kinematics and Physics of Celestial Bodies, 31, 13

Wu, Q., Yan, H., \& Yi, Z. 2013, MNRAS, 436, 1278

Yamauchi, A., Nakai, N., Ishihara, Y., Diamond, P., \& Sato, N. 2012, PASJ, 64 103

Yaqoob, T. 1997, ApJ, 479, 184

Yaqoob, T. 2012, MNRAS, 423, 3360

Zhu, G., Zaw, I., Blanton, M. R., \& Greenhill, L. J. 2011, ApJ, 742, 73 